\title{
Assortment Optimization under a Single Transition Model
}

\author{
Kameng Nip \\ Department of Mathematical Sciences, Tsinghua University, Beijing, China njm13@mails.tsinghua.edu.cn \\ Zhenbo Wang \\ Department of Mathematical Sciences, Tsinghua University, Beijing, China zwang@math.tsinghua.edu.cn \\ Zizhuo Wang \\ Department of Industrial and Systems Engineering, University of Minnesota, MN, USA zwang@umn.edu
}

\begin{abstract}
In this paper, we consider a Markov chain choice model with single transition. In this model, customers arrive at each product with a certain probability. If the arrived product is unavailable, then the seller can recommend a subset of available products to the customer and the customer will purchase one of the recommended products or choose not to purchase with certain transition probabilities. The distinguishing features of the model are that the seller can control which products to recommend depending on the arrived product and that each customer either purchases a product or leaves the market after one transition.

We study the assortment optimization problem under this model. Particularly, we show that this problem is generally NP-Hard even if each product could only transit to at most two products. Despite the complexity of the problem, we provide polynomial time algorithms for several special cases, such as when the transition probabilities are homogeneous with respect to the starting point, or when each product can only transit to one other product. We also provide a tight performance bound for revenue-ordered assortments. In addition, we propose a compact mixed integer program formulation that can solve this problem of large size. Through extensive numerical experiments, we show that the proposed algorithms can solve the problem efficiently and the obtained assortments could significantly improve the revenue of the seller than under the Markov chain choice model.
\end{abstract}

Key words: assortment optimization, choice model, mixed integer program, revenue-ordered assortment

\section{Introduction}

The fast development of information technology and rapid growth of online sales have presented great opportunities - as well as challenges - for retailers to use data to increase their bottom lines. One of the challenges is to determine which subset of products to make available to customers in order to maximize the expected revenue. Such a problem is often referred to as the assortment optimization problem. Lying in the heart of the assortment optimization problem is to identify an appropriate model to characterize the choice behavior of consumers when facing a subset of products. Much recent research has focused on finding such a choice model and solving the associated 
assortment optimization problem.

One of the consumer choice models that has recently gained much attention is the Markov chain choice model proposed by Blanchet et al. (2016). In the Markov chain choice model, it is assumed that customers arrive at each of the products with a certain exogenous probability. If the product a customer arrived at is unavailable (not in the offered assortment), then the customer will transit to other products (including the no-purchase option) with certain transition probabilities. And this process stops until the customer finds an available product to purchase or leaves the market. Many follow-up works have appeared based on the Markov chain choice model. We will provide a more detailed literature review in Section 2.

Although the Markov chain choice model brings a new perspective in modeling customer choice and has been validated by empirical data, it has two potential drawbacks. First, in the Markov chain choice model, it is assumed that customer's transition between products follows an exogenous process (according to the transition probabilities). However, in practice, after a customer's arrival, it is often the seller's recommendation that will determine the transition of customers. This is especially true in an online environment, in which customer's transition usually occurs when they click a product displayed in the recommendation section associated with an unavailable product. Second, in the Markov chain choice model, it is implicitly assumed that customers could transit arbitrarily many times among unavailable products, whereas in practice, customers often have much less patience and if a product transited to is unavailable, a customer may just leave the market altogether. This is especially likely in the situation when the transited product is a recommended product - in such a case, it is not hard to imagine that customers may become frustrated and as a result, abandon the entire purchase session.

In this paper, motivated to overcome the above two drawbacks in the Markov chain choice model, we propose a new choice model which we call the Markov Chain choice model with Single Transition, or MCST in short. Particularly, in the MCST model, customers initially arrive at each product with a certain probability as in the Markov chain choice model. However, if the product a customer arrived at is not available, under the MCST model, the seller can choose a subset of available products to recommend to the customer, and the customer will transit to each product in the recommended set (as well as the no-purchase option) based on a set of transition probabilities. Note that under the MCST model, a customer will either purchase a product or leave the market after a single transition. We believe the MCST model exhibits the following advantages compared to the Markov chain choice model:

1. Allow more flexible control for the seller in terms of which set of products to recommend after a customer searches into an unavailable product, which may give opportunities to achieve better revenue; 
2. By requiring that the seller can only recommend available products, the transition of customers is more realistic, which better reflects the limited patience of customers.

In this paper, we study the properties of the MCST model and the associated assortment optimization problem. In particular, we make the following theoretical contributions:

1. When the transition probabilities are homogeneous with respect to the starting points, we show that the optimal assortment under the MCST model is equivalent to that in the Markov chain choice model. In addition, we show that a revenue-ordered assortment must be optimal in this case and it can be solved in linear time of the number of products;

2. When each product can only transit to one other product, we show that the optimal assortment optimization problem under the MCST model can be solved in polynomial time by a dynamic program;

3. When a product could transit to more than one product, we show that the assortment optimization problem under the MCST model is generally NP-Hard. Nevertheless, we establish a tight performance bound for the revenue-ordered assortment in this case;

4. We establish a compact mixed integer program (MIP) formulation for the optimal assortment optimization problem under the MCST model. The MIP can be solved efficiently for large size problems thus is practically useful.

In addition to the theoretical results, we also conduct extensive numerical experiments for the MCST model and compare the performance with the Markov chain choice model and a recently proposed two product nonparametric choice model Paul et al. (2016). In particular, we show that under the same set of arrival and transition probabilities, it is more often that the optimal assortment under the MCST model achieves larger revenue than that under the Markov chain choice model or the two product nonparametric model, showing that the additional control of the seller over the recommended set is valuable. We also show that the optimal assortment solved from the MCST model is more robust under model misspecification situations.

As discussed in the beginning, sellers nowadays strive to utilize all possible data and control to improve their sales decisions and ultimately increase their bottom lines. The models and solutions proposed in this paper help the seller achieve this goal by allowing them to exert more controls in the customer choice process and helping them obtain better decisions. Therefore, we believe that our paper is helpful for sellers to meet the challenges presented in this age.

The remainder of this paper is organized as follows: In Section 2, we review related literature to our work. In Section 3, we formally state the problem studied in this paper. In Section 4, we study the problem where the transition weights are homogeneous with respect to the starting point. In Section 5, we investigate the heterogeneous case. We first study a special case, and then consider the computational complexity and algorithms for the general case. We present some numerical experiment results in Section 6. Finally, we provide some concluding remarks in Section 7. 


\section{Related Work}

In this section, we review related literature to our work. At the high level, our work is related to the fast growing research area of choice models and the associated revenue management problems. Particularly, our work is closely related to the recently proposed Markov chain choice model Blanchet et al. (2016) and the associated assortment optimization problem. In the following, we focus our literature review on these lines of works and refer the readers to Train (2009) and Özer and Phillips (2012) for comprehensive reviews for general research about choice models and revenue management respectively.

The Markov chain choice model is first introduced by Blanchet et al. (2016). In the Markov chain choice model, there is a line of products and each customer arrives at each product with a certain probability. If the product is available, then the customer will purchase it, otherwise the customer will transit to another product or leave with certain transition probabilities. The process terminates until the customer purchases a certain product or chooses to leave. In Blanchet et al. (2016), the authors show that such a model is a good approximation to several well-studied choice models. Moreover, they show that the assortment optimization problem under the Markov chain choice model can be solved in polynomial time by relating it to an optimal stopping problem on a Markov chain. There are several follow-up works that study the Markov chain choice model. For example, Feldman and Topaloglu (2014) study the network revenue management problem under the Markov chain choice model and present an algorithm based on linear optimization. Désir et al. (2016) study the capacity constrained assortment optimization problem. And Desir et al. (2015) study the robust assortment optimization under this model. As described in the introduction, the MCST model we propose modifies the Markov chain choice model in two important dimensions: 1) we add a control for the seller to decide which set of products to recommend when customers arrive at an unavailable product and 2) we only allow a single transition of the customers. We propose solution methods for solving the assortment optimization problem under the modified model and show that it has the potential to increase the revenue for the seller compared to that in the Markov chain choice model.

Our work is also related to the growing literature that studies assortment optimization problem. Assortment optimization is a central problem in revenue management in which the seller selects a subset of products to offer in order to maximize the expected revenue. The assortment optimization problem was first considered by Talluri and van Ryzin (2004), in which the authors study a single resource revenue management problem. They show that a class of revenue-ordered assortment is optimal when customers choose according to a multinomial logit (MNL) choice model. There are many subsequent works studying the assortment optimization problem under various consumer choice models. Some examples include the nested logit model (see, e.g., Li and Rusmevichientong 
2014, Davis et al. 2014, Gallego and Topaloglu 2014, Li et al. 2015); the mixture of multinomial logit (MMNL) model (see, e.g., Desir and Goyal 2014, Rusmevichientong et al. 2014), the rankingbased models (see, e.g., Honhon et al. 2012, Aouad et al. 2016, Bertsimas and Mišić 2016), the Mallows model (see e.g., Desir et al. 2016b, Desir et al. 2016a) and more recently, more complicated choice models such as the consider-then-choose model Aouad et al. (2015a), the MNL model with endogenous network effects Wang and Wang (2016), choice model when consumer searches for product information Sahin and Wang (2016), etc. In another direction, there has also been interest in studying assortment optimization problems under nonparametric choice models (see Farias et al. 2013, Jagabathula 2016, ?) or in a dynamic environment (see Rusmevichientong et al. 2010, ?, ?, Aouad et al. 2015b). In this work, we consider the assortment optimization problem under a new model, the MCST model. Similar to other works that study the assortment optimization problem, we study the complexity of the problem under different cases and propose efficient algorithms to solve the problem. We further compare the solution and the achieved revenue under our model with several related models.

In the study of assortment optimization problem, an important class of assortment is called the revenue-ordered assortment, in which the assortment is selected according to the revenue order of each product. Revenue-ordered assortment has been shown to be optimal in many assortment optimization problems or has a worst-case performance guarantee (see, e.g., Talluri and van Ryzin 2004, Rusmevichientong and Topaloglu 2012, Rusmevichientong et al. 2014, Wang and Wang 2016). Indeed, as shown in Berbeglia and Joret (2015), the optimal revenueordered assortment achieves a worst-case ratio of the optimal revenue under a class of "regular discrete choice models". In this paper, we show that the revenue-ordered assortment is optimal under a special case of the MCST model, and has a worst-case performance guarantee under the general case. Furthermore, in our numerical experiments, we show that the revenue-ordered assortment generally achieves good performance.

One closely related work to ours is the work by Paul et al. (2016). In Paul et al. (2016), the authors consider a two step choice model and a two product nonparametric choice model. Both of those models are based on the Markov chain choice model and only allow customers to make a single transition. In particular, the former one only allows homogeneous transition probabilities with respect to the starting point, while the latter one allows heterogeneous transition probabilities. They study the assortment optimization problem under these models, showing that both problems are NP-Hard, while proposing a fully polynomial time approximation scheme (FPTAS) for the former problem and a 2-approximation algorithm for the latter problem. Similar models have also been considered in Kök and Fisher (2007) in which the authors mainly consider the estimation problem and propose heuristic algorithms for the assortment optimization problem. In contrast 
to those models, in the MCST model, we allow the seller to choose which subset of products to recommend after a customer reaches an unavailable product instead of assuming the customer transitions are exogenous. As we will show in our paper, such flexibility will lead to a different optimization model for the assortment optimization problem and the achieved revenue could be much higher under the MCST model than in their models. Therefore, one can view our MCST model as a modification to the models in Paul et al. (2016), which could lead to significant revenue improvement for the sellers.

\section{Model}

In this section, we introduce the MCST model (Markov chain choice model with single transition) and the associated assortment optimization problem. Consider a seller who manages a collection of $n$ products denoted by $\mathcal{N}=\{1, \ldots, n\}$ with 0 being the no-purchase option. We assume each product $j$ has a fixed revenue $r_{j}$ (one can also view $r_{j}$ as the profit margin of product $j$ if the objective is to maximize profit). Without loss of generality, we assume that the products are sorted in the non-increasing order based on the revenue, i.e., $r_{1} \geq r_{2} \geq \cdots \geq r_{n}$. In the MCST model, the seller first selects an assortment $S \subseteq \mathcal{N}$ to offer to the customers (in other words, the seller makes available a subset of products $S$ ). Then, with probability $\lambda_{j} \geq 0$, a customer arrives at product $j$. (Naturally, we assume that $\sum_{j=1}^{n} \lambda_{j}=1$.) When a customer arrives at product $j$, if product $j$ is in the offered assortment $S$, then the customer purchases product $j$ and the seller gains revenue $r_{j}$. Otherwise, the seller can recommend a subset of products within the assortment, i.e., a subset $R_{j} \subseteq S$, and the customer will either select one of the products in $R_{j}$ or leave the market without purchasing any product. In particular, there is a weight $v_{j i} \geq 0$ for any $j \in \mathcal{N}$ and $i \in \mathcal{N} \cup\{0\}$, satisfying $\sum_{i \in \mathcal{N} \cup\{0\}} v_{j i}=1$ for any $j$, such that the probability a customer will transit to product $i \in R_{j}$ or leave the market (transit to product $i=0$ ) given that the customer arrived at product $j \notin S$ is

$$
\rho_{j i}=\frac{v_{j i}}{\sum_{i \in R_{j}} v_{j i}+v_{j 0}} .
$$

The assortment optimization problem under the MCST model (we will call it the MCST problem in the following) is to find an assortment $S \subseteq \mathcal{N}$, and for each $j \notin S$, a recommended subset of products $R_{j} \subseteq S$, such that the expected total revenue is maximized.

Now we derive the formal expression of the MCST problem. Let $\operatorname{Pr}_{i}\left(S,\left\{R_{j}\right\}_{j \notin S}\right)$ be the probability that $i$ is purchased by the customer when the offered assortment is $S$ and the recommended set for $j \notin S$ is $R_{j}$. For simplicity of notation, we denote the probability by $\operatorname{Pr}_{i}\left(S, R_{j}\right)$ when there is no confusion. We have 


$$
\operatorname{Pr}_{i}\left(S, R_{j}\right)= \begin{cases}\lambda_{i}+\sum_{j: j \notin S, i \in R_{j}} \lambda_{j} \frac{v_{j i}}{\sum_{i \in R_{j}} v_{j i}+v_{j 0}}, & \text { if } i \in S, \\ \sum_{j: j \notin S} \lambda_{j} \frac{v_{j 0}}{\sum_{i \in R_{j}} v_{j i}+v_{j 0}}, & \text { if } i=0, \\ 0, & \text { otherwise. }\end{cases}
$$

Let $\mathbf{R}\left(S, R_{j}\right)$ be the expected revenue when the offered assortment is $S$ and the recommended set for $j \notin S$ is $R_{j}$. Then we have

$$
\mathbf{R}\left(S, R_{j}\right)=\sum_{i \in S} r_{i} \operatorname{Pr}_{i}\left(S, R_{j}\right)=\sum_{i \in S} \lambda_{i} r_{i}+\sum_{j \notin S} \sum_{i \in R_{j}} \lambda_{j} r_{i} \frac{v_{j i}}{\sum_{i \in R_{j}} v_{j i}+v_{j 0}}
$$

and the assortment optimization problem under the MCST model can be written as

$$
\max _{S \subseteq \mathcal{N}, R_{j} \subseteq S, \forall j \notin S} \mathbf{R}\left(S, R_{j}\right)=\max _{S \subseteq \mathcal{N}, R_{j} \subseteq S, \forall j \notin S} \sum_{i \in S} \lambda_{i} r_{i}+\sum_{j \notin S} \sum_{i \in R_{j}} \lambda_{j} r_{i} \frac{v_{j i}}{\sum_{i \in R_{j}} v_{j i}+v_{j 0}} .
$$

We remark that in the case of $v_{j 0}=0$ for some $j \in S$, if the seller recommends none of the products (i.e., $R_{j}=\phi$ ), or only recommends products with zero transition weights $\left(v_{j i}=0\right)$, then the ratio in (2) would become $0 / 0$. To avoid confusion in those cases, we stipulate that $0 / 0=0$ in the rest of our discussion. Note that this is equivalent as setting the transition weight to the no-purchase option to an infinitesimal value when it is zero.

By examining (2), if we have chosen an assortment $S$, then the recommended set for any $j \notin S$ can be found by solving $\max _{R_{j} \subseteq S} \frac{\sum_{i \in R_{j}} r_{i} v_{j i}}{\sum_{i \in R_{j}} v_{j i}+v_{j 0}}$. Note that this is an assortment optimization problem under an attraction model, for which it has been shown that a revenue-ordered assortment is optimal Talluri and van Ryzin (2004). Therefore, we can efficiently find the optimal recommended set for each $j \notin S$ if the assortment $S$ has been determined. However, finding an optimal $S$ to offer initially is still a difficult task. As we will show in Section 5, the problem is NP-Hard in general, and we will develop algorithms for solving this problem in the following sections.

Before we end this section, we comment on the relation between the MCST model and the Markov chain choice model proposed in Blanchet et al. (2016) and the two step choice model and the two product nonparametric choice model proposed in Paul et al. (2016), which are all based on similar "transition" ideas. Compared to those models, a distinctive feature of the MCST model is that it allows the seller to choose a subset of products to recommend when a customer arrives at an unavailable product, and the subset chosen could depend on the product the customer arrived at. In other words, we allow the seller to control the transition of customers to some degree rather than assuming the transition is entirely exogenous. This is very reasonable since in practice, especially in the growing industry of online retailing, it is often the seller's recommendation that will determine the transition of the customers. Moreover, we only allow the seller to recommend products that are in the offered assortment, that is, we do not allow transition into unavailable products. This is 
also very reasonable as it does not make much sense in practice to recommend products that are unavailable (which will make customers frustrated or even annoyed and thus abandon the purchase session). Given the latter restriction, customer can only transit once in the MCST model rather than arbitrary number of times as in the Markov chain choice model. ${ }^{1}$ Later we will show that these features of the MCST model will lead to potentially different optimal assortment for the seller and also higher revenue.

\section{Homogeneous Case}

In this section, we first consider a special case of the MCST problem in which the transition probabilities of customers are homogeneous with respect to the starting product. More precisely, we consider the case in which there exists $v_{i} \geq 0$ for $i \in \mathcal{N} \cup\{0\}$ satisfying $\sum_{i \in \mathcal{N} \cup\{0\}} v_{i}=1$ such that $v_{j i}=v_{i}$ for all $i \in \mathcal{N} \cup\{0\}$ and $j \in \mathcal{N}$. Note that this condition ensures that the transition probability to a product $i$ given a recommended set $R$ is independent of the previous (unavailable) product $j$ (we will call $j$ the "starting point" in the following discussion). In the following, we will show that the optimal assortment under the homogeneous case of the MCST problem (which we will call the homogeneous MCST problem in the following) is identical to that under the Markov chain choice model with the same parameters, has a revenue-ordered structure, and can be solved very efficiently (in linear time of the number of products).

To start with, we note that in the homogeneous case, since the transition probabilities are homogeneous with respect to the starting point, there exists an optimal solution in which the optimal recommended sets $R_{j}$ s are identical for all $j \notin S$. The following theorem further shows that in this case, there is an optimal solution in which the seller recommends all the products in the offered sets:

Lemma 1. For the homogeneous MCST problem, given any assortment $S$, let $S^{\prime} \in$ $\arg \max _{R \subseteq S} \frac{\sum_{i \in R} r_{i} v_{i}}{\sum_{i \in R} v_{i}+v_{0}}$ be an optimal recommended set corresponding to $S$ such that there does not exist $S^{\prime \prime} \in \arg \max _{R \subseteq S} \frac{\sum_{i \in R} r_{i} v_{i}}{\sum_{i \in R} v_{i}+v_{0}}$ satisfying $S^{\prime} \varsubsetneqq S^{\prime \prime}$. Then $\mathbf{R}\left(S, S^{\prime}\right) \leq \mathbf{R}\left(S^{\prime}, S^{\prime}\right)$.

Remark: The condition that there does not exist $S^{\prime \prime} \in \arg \max _{R \subseteq S} \frac{\sum_{i \in R} r_{i} v_{i}}{\sum_{i \in R} v_{i}+v_{0}}$ such that $S^{\prime} \varsubsetneqq S^{\prime \prime}$ in Lemma 1 is necessary (in some sense, this condition can be understood as when there are several recommended sets that achieve the same revenue, we shall select the largest one). Otherwise, there could be a $k$ with $v_{k}=0$ and $r_{k}$ very large such that even though $S^{\prime}$ is an optimal recommended set with respect to $S=S^{\prime} \cup\{k\}, \mathbf{R}\left(S, S^{\prime}\right)>\mathbf{R}\left(S^{\prime}, S^{\prime}\right)$.

\footnotetext{
${ }^{1}$ It can be easily shown that if one first assumes that customers can only make a single transition, then it must be optimal for the seller to recommend products that are in the offered set. Therefore, the assumption that the seller only recommends products in the offered set is equivalent to the assumption that customers can only make a single transition. And the MCST model can be obtained through either assumption.
} 
The proofs of this and all subsequent lemmas/theorems are provided in the Appendix. Lemma 1 implies that there exists an optimal assortment $S^{*}$ to the homogeneous MCST problem, such that when we choose $S^{*}$ to offer, we recommend the same set $S^{*}$ for all $j \notin S^{*}$. Next we show that solving the homogeneous MCST problem is equivalent as solving the assortment optimization problem under the Markov chain choice model with the same parameters.

THEOREM 1. Consider the homogeneous MCST model and the Markov chain choice model with the same arrival probabilities $\lambda_{i}$ and transition probabilities $v_{j i}=v_{i}$. If $S^{*}$ is an optimal assortment under the Markov chain choice model, then offering $S^{*}$ and recommending $S^{*}$ for each $j \notin S^{*}$ is an optimal solution to the homogeneous MCST problem. Furthermore, the optimal values are the same.

Lemma 1 and Theorem 1 suggest that we can find the optimal assortment in the homogeneous MCST problem by solving the assortment optimization problem under the Markov chain choice model. It is known that the latter problem can be solved in polynomial time by relating to an optimal stopping problem on a Markov chain (Blanchet et al. 2016) or solving a linear optimization problem (Feldman and Topaloglu 2014). Thus the homogeneous MCST problem can be solved in polynomial time. In the following, we present an iterative algorithm for solving this problem which runs in linear time in the number of products, which is significantly faster than existing methods for solving the optimal assortment under the Markov chain choice model. The algorithm is motivated by the local ratio framework for the capacity constrained assortment optimization problem under the Markov chain choice model in Désir et al. (2016). Furthermore, we show by the iterative algorithm that a revenue-ordered assortment is optimal for the homogeneous MCST problem.

Recall that the products are sorted by non-increasing order based on their revenues. The iterative algorithm is described as follows: If $r_{1}<0$, then it is obvious that an empty assortment is optimal. Otherwise, we select product 1 into the optimal assortment. Then, we construct a new instance of an assortment optimization problem excluding product 1. Specifically, we keep the arrival probabilities $\lambda_{i} \mathrm{~s}$ unchanged and update the revenues and transition probabilities by setting

$$
r_{i}^{(1)}:=r_{i}-\frac{r_{1} v_{1}}{v_{1}+v_{0}}, \quad v_{i}^{(1)}=\left\{\begin{array}{ll}
v_{0}+v_{1} & \text { if } i=0 \\
v_{i} & \text { otherwise }
\end{array} \quad \text { for all } i \in \mathcal{N} \backslash\{1\} .\right.
$$

Let $\mathbf{R}^{(1)}(S, R)$ be the revenue of assortment $S$ under the updated revenue $r_{i}^{(1)}$ s and transition probabilities $v_{i}^{(1)} \mathrm{s}$, and when the recommended set for unavailable products is $R$. We have the following lemma:

Lemma 2. For any $S \subseteq \mathcal{N} \backslash\{1\}, \mathbf{R}(S \cup\{1\}, S \cup\{1\})=\mathbf{R}(\{1\},\{1\})+\mathbf{R}^{(1)}(S, S)$. Furthermore, when $r_{1} \geq 0, \mathbf{R}(S \cup\{1\}, S \cup\{1\}) \geq \mathbf{R}(S, S)$. 
According to Lemma 2, when $r_{1} \geq 0$, the optimal assortment to the homogeneous MCST problem must include product 1. Moreover, the optimal assortment would consist of product 1 and the optimal assortment to the updated instance with parameters $r_{i}^{(1)}$ and $v_{i}^{(1)}$. To find the optimal assortment of the updated instance, we can apply the same idea: We first check if $r_{2}^{(1)} \geq 0$. If so, we include product 2 and further update the instance (by computing $r_{i}^{(2)}$ and $v_{i}^{(2)}$ ); otherwise if $r_{2}^{(1)}<0$, then the optimal assortment to the updated instance must be an empty set. We can repeat the procedure until there is no positive revenue product in the updated instance and the assortment at that moment will be the optimal assortment of the MCST problem.

In Algorithm 1, we summarize the above procedure. The theorem below it shows that Algorithm 1 solves the homogeneous MCST problem in $O(n)$ time, which is significantly faster than solving an optimal stopping problem as in Blanchet et al. (2016) or solving an LP as in Feldman and Topaloglu (2014).

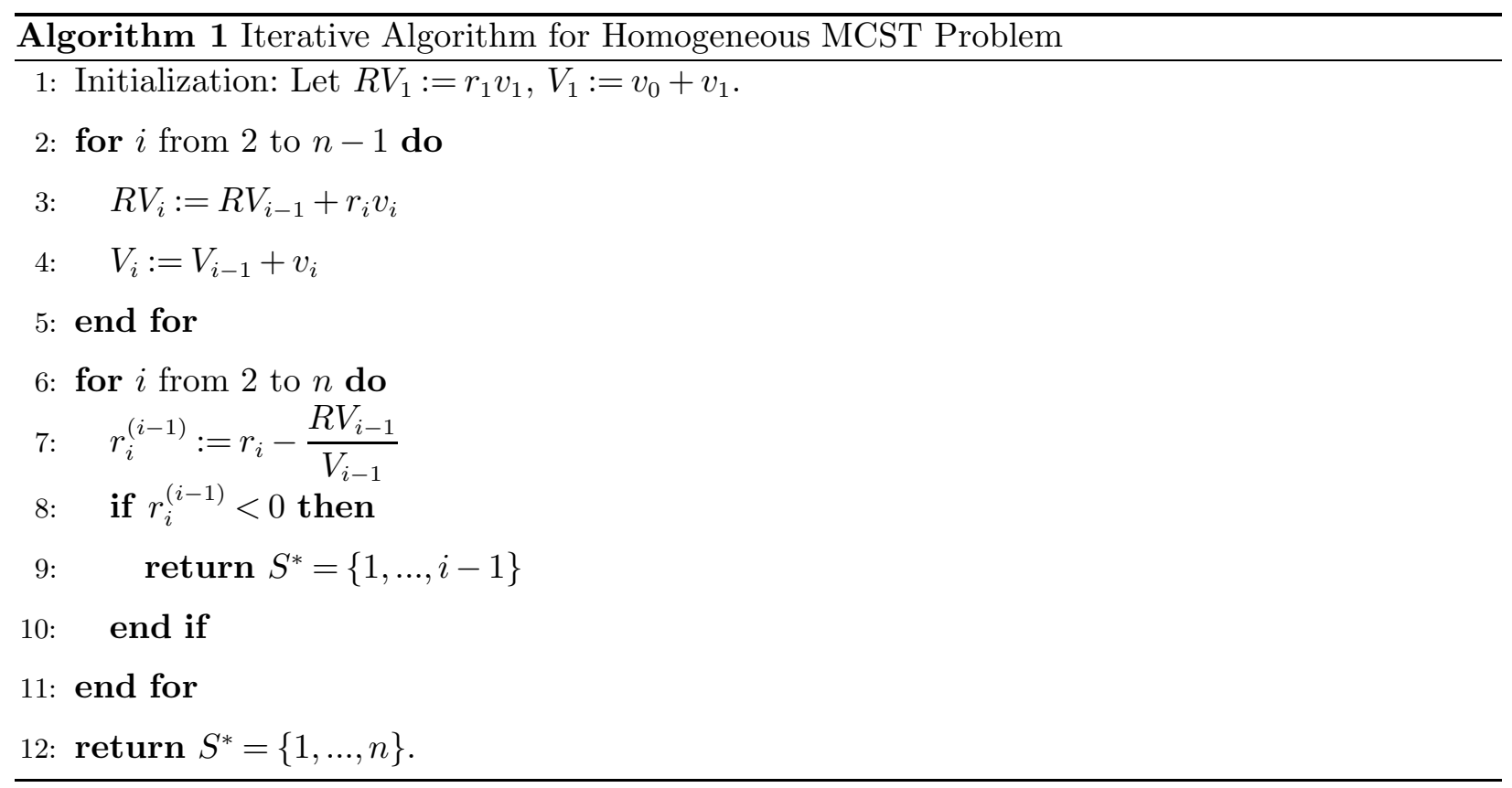

TheOREm 2. Algorithm 1 finishes in $O(n)$ time and the returned $S^{*}$ is an optimal assortment for the homogeneous MCST problem.

We observe that Algorithm 1 always returns a revenue-ordered assortment when it finishes, thus we have the following corollary.

COROLlARY 1. There is a revenue-ordered assortment that is optimal to the homogeneous MCST problem. 
In addition to obtaining efficient algorithms for solving the homogeneous MCST problem, by Theorems 1, 2 and Corollary 1, we know that for the Markov chain choice model, if the transition probabilities are homogenous with respect to the starting point, then a revenue-ordered assortment is optimal for the assortment optimization problem. Furthermore, there exists an algorithm that can solve the problem in linear time. As far as we know, this is the first time such a result is obtained which could also contribute to the study of the Markov chain choice model.

\section{Heterogeneous Case}

In this section, we consider the general MCST problem in which that transition probabilities are not necessarily homogeneous with respect to the starting point. We study the computational complexity of this problem as well as propose efficient algorithms for solving it (both in special cases as well as in general cases). We start with the following theorem stating that the problem is hard to solve in general.

THEOREM 3. The MCST problem is strongly NP-Hard, even if we restrict that each product can only transit to at most two products.

The proof of Theorem 3 is based on a reduction from the independent set problem and is relegated to the Appendix. By Theorem 3, it is not possible to obtain polynomial time algorithms for solving the MCST problem in general unless $P=N P$. Furthermore, by the strong NP-Hardness, it is unlikely that there is an FPTAS for the MCST problem either Gary and Johnson (1979). In the following, we first propose an efficient algorithm to solve the problem when each product can only transit to one product (Section 5.1), then establish a worst-case performance bound for the best revenue-ordered assortment (Section 5.2), and finally propose a compact mixed integer program (MIP) formulation that can solve the problem of practical size efficiently.

\subsection{Transit to One Product}

In this section, we consider a special case of the MCST problem in which each product can only transit to one other product (in addition to the no-purchase option). That is, for each $j \in \mathcal{N}$, there is at most one $i \in \mathcal{N}$ such that $v_{j i}>0$ (in addition to $v_{j 0}$ ). We show that the optimal assortment in this case can be found by solving a dynamic program in a linear time of the number of products.

In the following, if $v_{j i}>0$, then we say there is a link from $j$ to $i$. First, if there is a link from $j$ to $k$ with $r_{j} \geq r_{k}$, then it is easy to see that it is always optimal to include $j$ in the assortment and the link would become useless and thus can be safely removed. Thus, we can assume that all links from $j$ to $k$ are such that $j>k$, i.e., $r_{j}<r_{k}$. Therefore, we can construct a directed tree to indicate the transition relationship between the products (an example of such a tree is given in Figure 1). 
If there is a link from $j$ to $k$, then we call $j$ a child of $k$. Without loss of generality, we assume that product 1 is the root of the tree.

In the following, we show that we can solve the assortment optimization problem in this case by solving a dynamic program. Let $\mathcal{V}(i, 1)$ denote the optimal revenue that can be obtained using the products in the subtree starting from product $i$ and product $i$ is included in the assortment, and $\mathcal{V}(i, 0)$ denote the optimal revenue that can be obtained using the products in the subtree starting from product $i$ and product $i$ is not included in the assortment. We have the following recursion formula:

$$
\left\{\begin{array}{l}
\mathcal{V}(i, 1)=\lambda_{i} r_{i}+\sum_{\{j: j \text { is a child of } i\}} \max \left\{\mathcal{V}(j, 1), \frac{\lambda_{j} v_{j i} r_{i}}{v_{j i}+v_{j 0}}+\mathcal{V}(j, 0)\right\} \\
\mathcal{V}(i, 0)=\sum_{\{j: j \text { is a child of } i\}} \mathcal{V}(j, 1)
\end{array} \quad \text {, if } i\right. \text { is not a leaf }
$$

and

$$
\mathcal{V}(i, 1)=\lambda_{i} r_{i}, \quad \mathcal{V}(i, 0)=0 \quad \text { if } i \text { is a leaf. }
$$

To explain, $\mathcal{V}(i, 1)$ first includes the revenue of product $i$, which is $\lambda_{i} r_{i}$. Then for each $j$ that is a child of $i$, there are two choices: 1$)$ include product $j$, which leads to $\mathcal{V}(j, 1)$ from that node and below or 2) do not include product $j$, then there is a revenue of $\frac{\lambda_{j} v_{j i} r_{i}}{v_{j i}+v_{j 0}}$ from the transition from $j$ to $i$ and a revenue of $\mathcal{V}(j, 0)$ from the nodes below $j$. For $\mathcal{V}(i, 0)$, it does not generate revenue from product $i$, and for each child $j$, the optimal solution must include $j$ (otherwise it won't generate any revenue because $i$ is not in the assortment). Thus $\mathcal{V}(i, 0)=\sum_{\{j: j \text { is a child of } i\}} \mathcal{V}(j, 1)$.

To find the optimal assortment, we can compute the value functions from the leaves up to the root. Eventually, $\mathcal{V}(1,1)$ gives the optimal value (e.g., in Figure 1, we can compute the value of $\mathcal{V}$ in the order of $6 \rightarrow 5 \rightarrow 4 \rightarrow 3 \rightarrow 2 \rightarrow 1$ ). We have the following theorem regarding the complexity of this method.

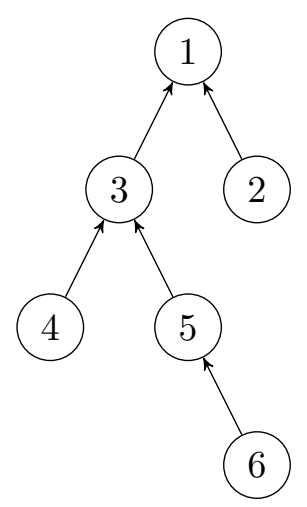

Figure 1 Example of the Tree 
THEOREM 4. If each product can transit to at most one other product in addition to the nopurchase option, then the MCST problem can be solved in $O(n)$ time by the dynamic program described above.

\subsection{Performance Bound of Revenue-Ordered Assortments}

In Section 4, we showed that a revenue-ordered assortment is optimal to the MCST problem in the homogeneous case. However, as shown in the beginning of this section, the MCST problem is NP-Hard in general, thus a revenue-ordered assortment is not necessarily optimal in general. In this section, we provide tight performance bound for revenue-ordered assortments for the MCST problem.

In some part, our discussion is motivated by the results in Berbeglia and Joret (2015). In Berbeglia and Joret (2015), the authors study the worst-case performance guarantee of the revenueordered assortments under a class of choice models, which they call general discrete choice model. Particularly, a choice model is said to be a general discrete choice model if it satisfies the following four conditions Berbeglia and Joret (2015):

1. $\operatorname{Pr}_{i}(S) \geq 0, \quad \forall i \in \mathcal{N} \cup\{0\}$ and $S \subseteq \mathcal{N}$;

2. $\operatorname{Pr}_{i}(S)=0, \quad \forall i \in \mathcal{N}$ and $S \subseteq \mathcal{N} \backslash\{i\}$;

3. $\sum_{i \in S} \operatorname{Pr}_{i}(S) \leq 1, \quad \forall S \subseteq \mathcal{N}$;

4. $\operatorname{Pr}_{i}(S) \leq \operatorname{Pr}_{i}\left(S^{\prime}\right), \quad \forall S^{\prime} \subseteq S \subseteq \mathcal{N}$ and $i \in S^{\prime} \cup\{0\}$.

The first three conditions are quite basic. The last condition, which is called the regularity axiom, says that if one shrinks the choice set, then the probability of choosing every alternative that remains in the choice set will increase. The following result is proved in Berbeglia and Joret (2015):

Theorem 5 (Theorems 3.1 and 3.2 in Berbeglia and Joret 2015). The best revenueordered assortment gives at least $\max \left\{\frac{1}{d}, \frac{1}{1+\log \frac{r_{\max }}{r_{\min }}}\right\}$ fraction of the optimal revenue under a general discrete choice model, where $d$ is the number of distinctive revenues among the alternatives and $r_{\max }$ and $r_{\min }$ are the highest and lowest revenues among the alternatives.

In the MCST model, we define the choice probability of product $i$ under set $S$ as the choice probability under $S$ with the optimal recommended sets for all $j \notin S$. That is, $\operatorname{Pr}_{i}(S)=\operatorname{Pr}_{i}\left(S, R_{j}\right)$ where $R_{j}=\arg \max _{R_{j} \subseteq S} \frac{\sum_{i \in R_{j}} r_{i} v_{j i}}{\sum_{i \in R_{j}} r_{i} v_{j i}+v j 0}$. In the following, for the ease of discussion, we assume that $R_{j}$ is unique for every $S$. (This can be achieved by adding an infinitesimal perturbation to the $v_{j i} \mathrm{~s}$.) The next example shows that the MCST model in general does not satisfy the regularity axiom, thus is not a general discrete choice model.

Example 1 We consider an example with four products with revenues $r_{1}=4, r_{2}=3, r_{3}=2$ and $r_{4}=1$. The arrival probabilities are $\lambda_{1}=\lambda_{2}=\lambda_{3}=\lambda_{4}=1 / 4$. The transition probabilities from 
product 1 to other products are $\left(v_{11}, v_{12}, v_{13}, v_{14}, v_{10}\right)=(0,0,0,1 / 2,1 / 2)$, and the transition probabilities from product 2 to other products are $\left(v_{21}, v_{22}, v_{23}, v_{24}, v_{20}\right)=(1 / 6,0,1 / 6,1 / 3,1 / 3)$. (We do not specify the transition probabilities for other pairs of products, as they are immaterial to the result.) Consider the assortments $S=(1,3,4)$ and $S^{\prime}=(3,4)$, and the choice probability of product 3. It can be calculated that the best recommended set for product 2 under $S$ is $(1,3)$, and the best recommended set for product 2 under $S^{\prime}$ is $(3,4)$. One can calculate that the choice probability $\operatorname{Pr}_{3}(S)=\lambda_{3}+\lambda_{2} \frac{v_{23}}{v_{21}+v_{23}+v_{20}}=0.3125$ is strictly greater than $\operatorname{Pr}_{3}\left(S^{\prime}\right)=\lambda_{3}+\lambda_{2} \frac{v_{23}}{v_{23}+v_{24}+v_{20}}=0.3$, which violates the regularity axiom.

Despite that the MCST model in general does not satisfy the definition of general discrete choice model thus we can't directly apply the results in Berbeglia and Joret (2015), we are still able to prove that the optimal revenue-ordered assortment achieves a certain fraction of the optimal revenue in the MCST problem. We have the following theorem:

THEOREM 6. The optimal revenue-ordered assortment gives at least $\max \left\{\frac{1}{d}, \frac{1}{1+\log \frac{r_{\max }}{r_{\min }}}\right\}$ fraction of the optimal revenue for the MCST problem. Furthermore, the performance bound is tight in that there exist instances such that the optimal revenue of the best revenue-ordered assortment is $O\left(\frac{1}{d}\right)$ or $O\left(\frac{1}{1+\log \frac{r_{\max }}{r_{\min }}}\right)$ fraction of the optimal revenue, respectively.

\subsection{Mixed Integer Programming Formulation}

In this section, we show that the MCST problem can be equivalently formulated as a mixed integer program (MIP). The MIP we formulate is very compact with only $n$ binary variables (the rest are continuous variables). As will be shown in Section 6, one can solve quite large size assortment optimization problem under the MCST model using this MIP formulation.

To obtain the MIP formulation, we define binary variables $x_{j}$ and $y_{j i}, \forall i, j=1, \ldots, n$, where $x_{j}=1$ indicates that product $j$ is selected in the assortment $S$, and $y_{j i}=1$ indicates that product $i$ is in the recommended set $R_{j}$ of product $j$. Then the optimization problem (2) can be formulated as the following nonlinear integer optimization problem:

$$
\begin{array}{lll}
\max & \sum_{j=1}^{n} \lambda_{j} r_{j} x_{j}+\sum_{j=1}^{n} \lambda_{j}\left(1-x_{j}\right) \frac{\sum_{i=1}^{n} r_{i} v_{j i} y_{j i}}{\sum_{i=1}^{n} v_{j i} y_{j i}+v_{j 0}} & \\
\text { s.t. } & y_{j i} \leq x_{i}, & \forall i, j=1, \ldots, n, \\
& y_{j i} \leq 1-x_{j}, & \forall i, j=1, \ldots, n, \\
& x_{i}, y_{j i} \in\{0,1\}, & \forall i, j=1, \ldots, n .
\end{array}
$$

In the above formulation, constraints (4b) and (4c) guarantee that a product $i$ can be recommended after product $j$ is visited only if product $j$ is not included in the assortment but product $i$ is. In 
the following, we assume that $v_{j 0} \neq 0$ for all $j \in \mathcal{N}$ (otherwise we can set $v_{j 0}$ to be an infinitesimal number, and all the results follow in the same way). Similar to the idea in Davis et al. (2013), we can rewrite (4) as the following mixed integer program:

$$
\begin{aligned}
& \max \sum_{j=1}^{n} \lambda_{j} r_{j} x_{j}+\sum_{j=1}^{n} \lambda_{j} \sum_{i=1}^{n} r_{i} z_{j i} \\
& \text { s.t. } z_{j i} \leq x_{i}, \quad \forall i, j=1, \ldots, n \text {, } \\
& \sum_{i=1}^{n} z_{j i}+z_{j 0}=1-x_{j}, \quad \forall j=1, \ldots, n, \\
& 0 \leq z_{j i} \leq \frac{v_{j i}}{v_{j 0}} z_{j 0}, \quad \forall i, j=1, \ldots, n, \\
& x_{i} \in\{0,1\}, \quad \forall i=1, \ldots, n .
\end{aligned}
$$

It can be easily seen that (5) is a relaxation for (4) (by noting that one can set $z_{j i}=(1-$ $\left.x_{j}\right) \frac{v_{j i} y_{j i}}{\sum_{i=1}^{n} v_{j i} y_{j i}+v_{j 0}}$ and $\left.z_{j 0}=\left(1-x_{j}\right) \frac{v_{j 0}}{\sum_{i=1}^{n} v_{j i} y_{j i}+v_{j 0}}\right)$. In the following, we show that the relaxation is exact. We have the following theorem, which uses similar idea as in Davis et al. (2013) but requires a different proof.

Theorem 7. If $\left(\boldsymbol{x}^{*}, \boldsymbol{y}^{*}\right)$ is an optimal solution to (4), then there exists $\boldsymbol{z}^{*}$ such that $\left(\boldsymbol{x}^{*}, \boldsymbol{z}^{*}\right)$ is an optimal solution to (5). Conversely, if $\left(\boldsymbol{x}^{*}, \boldsymbol{z}^{*}\right)$ is an optimal solution to (5), then there exists $\boldsymbol{y}^{*}$ such that $\left(\boldsymbol{x}^{*}, \boldsymbol{y}^{*}\right)$ is an optimal solution to (4). Furthermore, the optimal values to (4) and (5) are equal.

Theorem 7 essentially says that problem (4) is equivalent to problem (5). Thus in practice, one can solve (5) in order to solve the MCST problem. Note that there are only $n$ binary variables in (5). As will be shown in the next section, the MIP formulation is very efficient for solving the MCST problem, even for problems of practical size.

\section{Numerical Experiments}

In this section, we conduct numerical experiments to test the MCST model and the proposed algorithms. The objectives of the numerical experiments are mainly three fold: 1) demonstrate the efficiency of the MIP formulation for solving the MCST problem and test the performance of the revenue-ordered assortments, 2) illustrate the benefit of allowing the seller to choose different recommendation sets for different products and 3) study the robustness of the assortment decision of different models by considering several scenarios with model misspecification. Specifically, for the second and third parts, we will compare the performance of the optimal assortment under the MCST model with that under the Markov chain choice model Blanchet et al. (2016) and the two product nonparametric choice model Paul et al. (2016). 
We start by describing the setup of the experiments. In our experiments, we consider problem instances with $n \in\{5,10,30,50,100,300,500\}$ products in the first part and $n \in\{5,10,30,50\}$ products in the second and third parts (since solving large instances for the two product nonparametric choice model is challenging). Such a range should be enough to cover most practical scenarios. We consider the following ways to generate the parameters in the numerical experiments:

1. Revenue: The revenue of the products $r_{j} \mathrm{~s}$ are generated by first generating $n$ independent random numbers from uniform distributions on $[0,1]$ (exponential distributions with mean 1 , resp.) and then sorting them in decreasing order. We use UNI (EXP, resp.) to denote the corresponding way of generating the revenues.

2. Arrival and transition probabilities: For the arrival probabilities, we generate $n$ independent random numbers $\Lambda_{j}$, each from a uniform distribution on $[0,1]$, and set the arrival probabilities to be $\lambda_{j}=\Lambda_{j} / \sum_{j=1}^{n} \Lambda_{j}$ for all $j$. For the transition probabilities, we consider two methods of generating them. In the first method, for each $j$, we generate $n+1$ independent random numbers $\Lambda_{j i}$, each from a uniform distribution on $[0,1]$. Then we set $v_{j i}=\Lambda_{j i} / \sum_{i=0}^{n} \Lambda_{j i}$. We use DEN (meaning dense) to denote this method. In the second method, we make the transition matrix sparse to reflect that customer interested in one product typically is only interested in a few other products. Particularly, for each $\Lambda_{j i}$, with probability $1-1 / \sqrt{n}$, we set it to be zero and with probability $1 / \sqrt{n}$, we set it to be a uniformly distributed random number on $[0,1]$ (for $\Lambda_{j 0}$, we always set it to be a uniformly distributed random number on $[0,1]$, meaning that the customer can always transit to the no-purchase option). Then we set $v_{j i}=\Lambda_{j i} / \sum_{i=0}^{n} \Lambda_{j i}$. We use SPA (meaning sparse) to denote this method.

In the presentation of the results, we will use the notation $(n, \mathbf{A}, \mathbf{B})$ to denote the combination in which there are $n$ products, method A (one of UNI or EXP) is used to generate the revenues and method B (one of DEN or SPA) is used to generate the transition probabilities. All our numerical experiments are conducted on a PC with 4GB Memory and Intel(R) Core(TM) i5-4200U CPU (@1.6GHz) using MATLAB R2016a. And all optimization problems are solved using Gurobi 7.01 (Gurobi Optimization, Inc. 2016).

\subsection{Performance of the MIP and Revenue-Ordered Assortment}

In this subsection, we first test the performance of the MIP formulation proposed in Section 5.3 as well as the performance of revenue-ordered assortments. The results are shown in Table 1.

In Table 1, each number is calculated by averaging 1,000 test instances in the corresponding combination of parameters. The first and second columns show the average runtime (in seconds) of the MIP formulation and the average runtime for calculating the best revenue-ordered assortment, respectively. The third and fourth columns show the average and worst-case performance ratio 
between the best revenue-ordered assortment and the optimal assortment, respectively. From Table 1 , we can see that the MIP formulation is very efficient. Particularly, it can solve problems with up to 500 products within a matter of minutes, and for problems that have sparse structure, it is even more efficient. (In comparison, if one enumerates all possible assortments, then even for problems that have 30 products, it will take hours to solve.) For the revenue-ordered assortment, we can see that for the dense cases, it performs quite well, with average performance ratio being more than $99 \%$, and the performance is especially good when the problem size is large. However, for sparse problems, the revenue-ordered assortment performs less well, sometimes giving rise to large performance gaps. Therefore, given its efficiency, we suggest using the MIP formulation for practical problems.

\begin{tabular}{|c|c|c|c|c|}
\hline Parameters & MIP Time (s) & RO Time (s) & RO Ave. Ratio & RO Min. Ratio \\
\hline$(5, \mathbf{U N I}, \mathbf{D E N})$ & 0.0032 & 0.0003 & $99.72 \%$ & $86.57 \%$ \\
\hline$(10, \mathbf{U N I}, \mathbf{D E N})$ & 0.0064 & 0.0004 & $99.76 \%$ & $97.64 \%$ \\
\hline$(30, \mathbf{U N I}, \mathbf{D E N})$ & 0.0654 & 0.0005 & $99.93 \%$ & $99.25 \%$ \\
\hline$(50, \mathbf{U N I}, \mathbf{D E N})$ & 0.2184 & 0.0007 & $99.96 \%$ & $99.56 \%$ \\
\hline$(100, \mathbf{U N I}, \mathbf{D E N})$ & 1.1465 & 0.0012 & $99.98 \%$ & $99.75 \%$ \\
\hline$(300, \mathbf{U N I}, \mathbf{D E N})$ & 20.1697 & 0.0036 & $99.99 \%$ & $99.96 \%$ \\
\hline$(500, \mathbf{U N I}, \mathbf{D E N})$ & 70.0654 & 0.0082 & $99.99 \%$ & $99.98 \%$ \\
\hline$\overline{(5, \mathbf{E X P}, \mathbf{D E N})}$ & 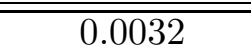 & $\overline{\overline{0.0002}}$ & 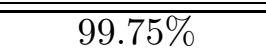 & $89.36 \%$ \\
\hline$(10, \mathbf{E X P}, \mathbf{D E N})$ & 0.0062 & 0.0003 & $99.71 \%$ & $93.58 \%$ \\
\hline$(30, \mathbf{E X P}, \mathbf{D E N})$ & 0.0538 & 0.0004 & $99.86 \%$ & $97.88 \%$ \\
\hline$(50, \mathbf{E X P}, \mathbf{D E N})$ & 0.1580 & 0.0005 & $99.91 \%$ & $98.15 \%$ \\
\hline$(100, \mathbf{E X P}, \mathbf{D E N})$ & 0.8802 & 0.0008 & $99.95 \%$ & $99.07 \%$ \\
\hline$(300, \mathbf{E X P}, \mathbf{D E N})$ & 11.3842 & 0.0021 & $99.98 \%$ & $99.79 \%$ \\
\hline$(500, \mathbf{E X P}, \mathbf{D E N})$ & 39.3100 & 0.0039 & $99.99 \%$ & $99.92 \%$ \\
\hline$\overline{(5, \text { UNI, SPA })}$ & $\overline{0.0034}$ & $\overline{0.0002}$ & $\overline{99.01 \%}$ & 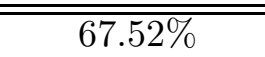 \\
\hline$(10, \mathbf{U N I}, \mathbf{S P A})$ & 0.0046 & 0.0004 & $98.44 \%$ & $88.35 \%$ \\
\hline$(30, \mathbf{U N I}, \mathbf{S P A})$ & 0.0144 & 0.0009 & $98.15 \%$ & $91.00 \%$ \\
\hline (50, UNI, SPA $)$ & 0.0380 & 0.0014 & $98.40 \%$ & $94.09 \%$ \\
\hline$(100, \mathbf{U N I}, \mathbf{S P A})$ & 0.1570 & 0.0031 & $98.51 \%$ & $96.86 \%$ \\
\hline$(300, \mathbf{U N I}, \mathbf{S P A})$ & 1.8557 & 0.0153 & $98.63 \%$ & $97.43 \%$ \\
\hline$(500, \mathbf{U N I}, \mathbf{S P A})$ & 6.8322 & 0.0418 & $98.68 \%$ & $97.73 \%$ \\
\hline$\overline{(\overline{(5, \mathbf{E X P}, \mathbf{S P A})}}$ & $\overline{0.0034}$ & $\overline{\overline{0.0002}}$ & 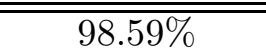 & $\overline{772.72 \%}$ \\
\hline$(10, \mathbf{E X P}, \mathbf{S P A})$ & 0.0047 & 0.0004 & $97.74 \%$ & $86.39 \%$ \\
\hline$(30, \mathbf{E X P}, \mathbf{S P A})$ & 0.0151 & 0.0008 & $96.59 \%$ & $86.73 \%$ \\
\hline$(50, \mathbf{E X P}, \mathbf{S P A})$ & 0.0412 & 0.0013 & $96.09 \%$ & $89.38 \%$ \\
\hline$(100, \mathbf{E X P}, \mathbf{S P A})$ & 0.1642 & 0.0027 & $96.65 \%$ & $93.09 \%$ \\
\hline$(300, \mathbf{E X P}, \mathbf{S P A})$ & 1.9177 & 0.0111 & $97.46 \%$ & $94.73 \%$ \\
\hline$(500, \mathbf{E X P}, \mathbf{S P A})$ & 6.6410 & 0.0243 & $97.86 \%$ & $96.43 \%$ \\
\hline
\end{tabular}

Table 1 Running Time and Performance of the MIP Formulation and Revenue-Ordered Assortments 


\subsection{The Benefit of Allowing Recommending Different Subsets}

In this section, we study the benefit of allowing the seller to recommend products based on the previously visited (unavailable) product. We will consider three models, the MCST model, the Markov chain choice model Blanchet et al. (2016) and the two product nonparametric model Paul et al. (2016). Note that these three models can be captured by the same set of parameters - the revenue of each product, the arrival probabilities, and the transition probabilities. In our numerical tests, we will generate instances according to the methods described in the beginning of Section 6 . For each instance, we will solve the optimal assortment under each model respectively, and calculate the optimal revenue under each model. Note that for the MCST model, we will use the MIP formulation in Section 5.3 to solve for the optimal assortment; for the Markov chain choice model, we will use the LP formulation as described in Feldman and Topaloglu (2014); and for the two product nonparametric model, we will solve the following binary quadratic program ${ }^{2}$ :

$$
\max _{\boldsymbol{x} \in\{0,1\}^{n}} \sum_{j=1}^{n} \lambda_{j} r_{j} x_{j}+\sum_{j=1}^{n} \sum_{i=1}^{n} \lambda_{i} r_{j} v_{i j}\left(1-x_{i}\right) x_{j} .
$$

The results are shown in Table 2. In Table 2, the first section shows the comparison between the MCST model and the Markov chain choice model. (In Table 2, we use the word Choosy to denote the two product nonparametric choice model as the authors of Paul et al. (2016) call this type of customers "choosy customers".) Particularly, the first subcolumn shows the percentage of times that the optimal value of the MCST model is larger than that of the Markov chain choice model among the 1,000 test instances. The second, third and fourth subcolumns show the average, minimum and maximum ratios between the optimal value of the MCST model and the optimal value of the Markov chain choice model among the 1,000 test instances, respectively. Similarly, the second section shows the comparison between the MCST model and the two product nonparametric choice model. Particularly, the first subcolumn shows the percentage of times that the optimal value of the MCST model is larger than that of the two product nonparametric choice model among the 1,000 test instances. The second, third and fourth subcolumns show the average, the minimum and the maximum ratios between the optimal value of the MCST model and the optimal value of the two product nonparametric choice model among the 1,000 test instances, respectively.

There are two forces that drive the comparison between the MCST and the Markov chain choice model. On the one hand, the MCST allows the seller to recommend different subset of products depending on the previously visited product, which would increase the optimal revenue. On the

\footnotetext{
${ }^{2}$ In Paul et al. (2016), the authors proposed efficient methods to solve the problem approximately. However, since we are comparing the optimal solution, we use the exact formulation. In our numerical tests, the binary quadratic program can be solved efficiently for $n$ up to 50 . However, for $n$ greater than 100, it would take several days to compute 1,000 instances. So we only report the results up to $n=50$.
} 


\begin{tabular}{|c|c|c|c|c|c|c|c|c|}
\hline \multirow[b]{2}{*}{ Parameters } & \multicolumn{4}{|c|}{ MCST/Markov } & \multicolumn{4}{|c|}{ MCST/Choosy } \\
\hline & $\begin{array}{l}\text { MCST } \geq \\
\text { Markov }\end{array}$ & $\begin{array}{l}\text { Ave. } \\
\text { Ratio }\end{array}$ & $\begin{array}{l}\text { Min. } \\
\text { Ratio }\end{array}$ & $\begin{array}{l}\text { Max. } \\
\text { Ratio }\end{array}$ & $\begin{array}{c}\text { MCST } \geq \\
\text { Choosy }\end{array}$ & $\begin{array}{l}\text { Ave. } \\
\text { Ratio }\end{array}$ & $\begin{array}{l}\text { Min. } \\
\text { Ratio }\end{array}$ & $\begin{array}{l}\text { Max. } \\
\text { Ratio }\end{array}$ \\
\hline$(5, \mathbf{U N I}, \mathbf{D E N})$ & $70.00 \%$ & 1.0123 & 0.7758 & 1.2405 & $100 \%$ & 1.1582 & 1.0000 & 2.5825 \\
\hline$(10, \mathbf{U N I}, \mathbf{D E N})$ & $75.80 \%$ & 1.0142 & 0.9384 & 1.2210 & $100 \%$ & 1.2350 & 1.0149 & 1.9259 \\
\hline$(30, \mathbf{U N I}, \mathbf{D E N})$ & $85.80 \%$ & 1.0098 & 0.9811 & 1.0494 & $100 \%$ & 1.3674 & 1.1456 & 1.8206 \\
\hline$(50, \mathbf{U N I}, \mathbf{D E N})$ & $93.20 \%$ & 1.0089 & 0.9906 & 1.0334 & $100 \%$ & 1.4268 & 1.2276 & 1.8000 \\
\hline$(5, \mathbf{E X P}, \mathbf{D E N})$ & $61.20 \%$ & 1.0152 & 0.7194 & $\bar{~} 1.4270$ & $100 \%$ & 1.3083 & 1.0000 & 3.0025 \\
\hline$(10, \mathbf{E X P}, \mathbf{D E N})$ & $69.90 \%$ & 1.0241 & 0.8158 & 1.2339 & $100 \%$ & 1.5497 & 1.0622 & 3.5257 \\
\hline$(30, \mathbf{E X P}, \mathbf{D E N})$ & $87.60 \%$ & 1.0316 & 0.8411 & 1.1890 & $100 \%$ & 2.0562 & 1.4099 & 3.5480 \\
\hline$(50, \mathbf{E X P}, \mathbf{D E N})$ & $93.40 \%$ & 1.0341 & 0.9181 & 1.1855 & $100 \%$ & 2.3341 & 1.5786 & 4.4266 \\
\hline 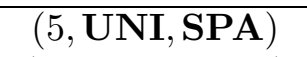 & $79.80 \%$ & 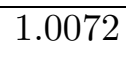 & 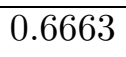 & 1.6459 & $100 \%$ & 1.0570 & $\bar{~} 1.0000$ & 1.9723 \\
\hline (10, UNI, SPA) & $62.50 \%$ & 1.0058 & 0.8305 & 1.2445 & $100 \%$ & 1.0770 & 1.0000 & 1.4575 \\
\hline (30, UNI, SPA) & $66.50 \%$ & 1.0078 & 0.9138 & 1.1038 & $100 \%$ & 1.1298 & 1.0167 & 1.3458 \\
\hline$(50, \mathbf{U N I}, \mathbf{S P A})$ & $65.20 \%$ & 1.0059 & 0.9570 & 1.0607 & $100 \%$ & 1.1573 & 1.0532 & 1.3249 \\
\hline 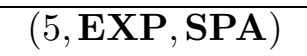 & $71.30 \%$ & 1.0097 & 0.6601 & 1.7145 & $100 \%$ & 1.1055 & $\bar{~} 1.0000$ & 2.2900 \\
\hline$(10, \mathbf{E X P}, \mathbf{S P A})$ & $56.20 \%$ & 1.0081 & 0.6320 & 1.3988 & $100 \%$ & 1.1582 & 1.0000 & 2.1188 \\
\hline$(30, \mathbf{E X P}, \mathbf{S P A})$ & $59.30 \%$ & 1.0138 & 0.7800 & 1.3191 & $100 \%$ & 1.3032 & 1.0879 & 2.0047 \\
\hline$(50, \mathbf{E X P}, \mathbf{S P A})$ & $61.80 \%$ & 1.0131 & 0.8690 & 1.2225 & $100 \%$ & 1.3809 & 1.1786 & 1.9767 \\
\hline
\end{tabular}

Table 2 Comparison between the MCST and Other Models

other hand, the MCST only allows a single transition of the customer, which would decrease the optimal revenue. As shown in Table 2, the two forces roughly balance on average in terms of the performance ratio, with MCST generating higher optimal value more frequently. Here we comment that the advantage of the MCST requires the seller to take action (of recommending different subsets) while the disadvantage is more of a modeling assumption. Particularly, customers who will transit multiple times will still transit multiple times in the MCST model, which will make the disadvantage less significant. This becomes more evident when we compare the MCST model and the two product nonparametric model. In this comparison, only the advantage of the MCST model exists (customers can only transit once under both models). From Table 2, we can see that the benefit of allowing the seller to recommend different subsets based on the starting point is significant, with an average of $10 \%-40 \%$ revenue increment in many parameter settings and over $130 \%$ in some instances. Therefore, the results indicate that allowing the seller to recommend different subset of products based on the arrival product could be a revenue driver in practice.

\subsection{Robustness of the Results}

In this section, we study the robustness of the assortment generated from the MCST model (as well as other models) by considering several model misspecification scenarios. Particularly, we investigate the loss of revenue if we use the optimal assortment solved from one model but the customer behaves according to another model. Since the optimal assortment from the MCST model 
is clearly the best if the underlying model is indeed the MCST model, we will mainly consider the cases in which the underlying model is either the Markov chain choice model or the two product nonparametric model.

\begin{tabular}{|c|c|c|c|c|c|c|c|c|}
\hline \multirow{3}{*}{ Parameters } & \multicolumn{4}{|c|}{ Average Ratio } & \multicolumn{4}{|c|}{ Minimum Ratio } \\
\hline & \multicolumn{2}{|c|}{ True Markov } & \multicolumn{2}{|c|}{ True Choosy } & \multicolumn{2}{|c|}{ True Markov } & \multicolumn{2}{|c|}{ True Choosy } \\
\hline & $\overline{\text { MCST }}$ & Choosy & $\overline{\mathrm{MCST}}$ & Markov & $\overline{\mathrm{MCST}}$ & Choosy & $\overline{\mathrm{MCST}}$ & Markov \\
\hline$(5, \mathbf{U N I}, \mathbf{D E N})$ & 0.9940 & 0.9408 & 0.9229 & 0.9087 & 0.7314 & 0.5245 & 0.4579 & 0.4219 \\
\hline$(10, \mathbf{U N I}, \mathbf{D E N})$ & 0.9933 & 0.8950 & 0.8437 & 0.8106 & 0.8139 & 0.6508 & 0.4740 & 0.3782 \\
\hline$(30, \mathbf{U N I}, \mathbf{D E N})$ & 0.9947 & 0.8160 & 0.6605 & 0.6048 & 0.9268 & 0.6392 & 0.3933 & 0.3068 \\
\hline$(50, \mathbf{U N I}, \mathbf{D E N})$ & 0.9953 & 0.7858 & 0.5738 & 0.5117 & 0.9554 & 0.6685 & 0.3118 & 0.2697 \\
\hline 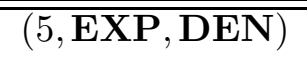 & 0.9875 & 0.8908 & 0.9129 & 0.8760 & 0.5986 & 0.4628 & 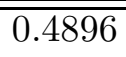 & 0.2620 \\
\hline$(10, \mathbf{E X P}, \mathbf{D E N})$ & 0.9755 & 0.7862 & 0.8015 & 0.7379 & 0.7135 & 0.3699 & 0.3989 & 0.2526 \\
\hline$(30, \mathbf{E X P}, \mathbf{D E N})$ & 0.9669 & 0.6170 & 0.5781 & 0.4744 & 0.7364 & 0.3601 & 0.2664 & 0.1434 \\
\hline$(50, \mathbf{E X P}, \mathbf{D E N})$ & 0.9660 & 0.5475 & 0.4734 & 0.3729 & 0.7039 & 0.3142 & 0.2633 & 0.0856 \\
\hline 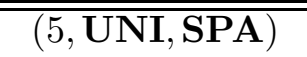 & 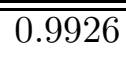 & "0.9799 & 0.9845 & 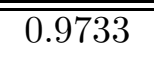 & 0.6568 & 0.6384 & 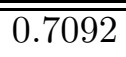 & 0.4048 \\
\hline (10, UNI, SPA) & 0.9860 & 0.9657 & 0.9670 & 0.9492 & 0.8242 & 0.7850 & 0.7073 & 0.6487 \\
\hline$(30, \mathbf{U N I}, \mathbf{S P A})$ & 0.9824 & 0.9350 & 0.9311 & 0.8953 & 0.8913 & 0.7735 & 0.7875 & 0.6723 \\
\hline$(50, \mathbf{U N I}, \mathbf{S P A})$ & 0.9830 & 0.9171 & 0.9083 & 0.8632 & 0.9184 & 0.8257 & 0.7884 & 0.6740 \\
\hline 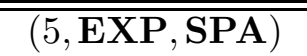 & 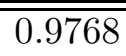 & 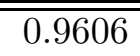 & 0.9785 & 0.9531 & 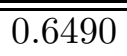 & 0.6500 & 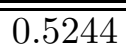 & 0.3588 \\
\hline$(10, \mathbf{E X P}, \mathbf{S P A})$ & 0.9593 & 0.9261 & 0.9673 & 0.9180 & 0.6489 & 0.5516 & 0.6971 & 0.4316 \\
\hline$(30, \mathbf{E X P}, \mathbf{S P A})$ & 0.9314 & 0.8540 & 0.9210 & 0.8224 & 0.6245 & 0.5817 & 0.7602 & 0.4510 \\
\hline$(50, \mathbf{E X P}, \mathbf{S P A})$ & 0.9244 & 0.8185 & 0.8940 & 0.7705 & 0.7179 & 0.6065 & 0.7424 & 0.3766 \\
\hline
\end{tabular}

Again, we will consider the experimental setups as described in the beginning of Section 6 . The results are shown in Table 3. In Table 3, we show the average and worst-case performance ratios when the underlying model is one model and one uses the optimal assortment solved by assuming another model. For example, the True Markov-MCST column under the Average Ratio section shows the average performance ratio of using the optimal assortment solved from the MCST model when the true underlying model is the Markov chain choice model versus using the optimal assortment of the true Markov model. From Table 3, we can see that our model is more robust than the other models. In particular, when the underlying model is misspecified, using the assortment solved from the MCST model usually results in better performance than using the third model. This suggests using the optimal assortment under the MCST model is a robust choice.

\section{Conclusion}

In this paper, we considered a Markov chain choice model with single transition. In particular, the seller can choose a recommendation set based on the visited product. We proposed algorithms for solving the assortment optimization problem under this model. We showed that this problem can 
be solved in polynomial time under several special cases and proposed an efficient MIP formulation for solving the general problem. We demonstrated via numerical experiments that the model has potential for increasing the revenue of the seller.

There are several future directions for research. First, theoretically, it would be interesting to see if there are other cases in which the assortment optimization problem under the MCST model can be solved in polynomial time. Second, it would be important to study the estimation problem under this model. Last, it would be very interesting to validate this model using real data and see how effective the optimal assortment of the MCST model is in practice.

\section{References}

Aouad, A., V. Farias, R. Levi, D. Segev. 2016. The approximability of assortment optimization under ranking preferences. Working Paper.

Aouad, A., V. F. Farias, R. Levi. 2015a. Assortment optimization under consider-then-choose choice models. Working Paper.

Aouad, Ali, Retsef Levi, Danny Segev. 2015b. Approximation algorithms for dynamic assortment optimization models. working paper .

Berbeglia, G., G. Joret. 2015. Assortment optimisation under a general discrete choice model: A tight analysis of revenue-ordered assortments. Working Paper.

Bertsimas, D., V. Mišić. 2016. Data-driven assortment optimization. Working Paper.

Blanchet, J., G. Gallego, V. Goyal. 2016. A Markov chain approximation to choice modeling. Operations Research 64(4) 886-905.

Davis, J., G. Gallego, H. Topaloglu. 2013. Assortment planning under the multinomial logitmodelwith totally unimodular constraint structures. Working Paper.

Davis, J. M., G. Gallego, H. Topaloglu. 2014. Assortment optimization under variants of the nested logit model. Operations Research 62(2) 250-273.

Desir, A., V. Goyal. 2014. Near-optimal algorithms for capacity constrained assortment optimization. Working paper.

Desir, A., V. Goyal, S. Jagabathula, D. Segev. 2016a. Assortment optimization under a mixture of Mallows model. Working Paper.

Desir, A., V. Goyal, S. Jagabathula, D. Segev. 2016b. Assortment optimization under the Mallows model. NIPS'16: Advances in Neural Information Processing Systems. 4700-4708.

Désir, A., V. Goyal, D. Segev, C. Ye. 2016. Capacity constrained assortment optimization under the Markov chain based choice model. Operations Research Forthcoming. 
Desir, A., V. Goyal, H. Topalogu, J. Zhang. 2015. Robust assortment optimization under the markov chain model. Working Paper.

Farias, V. F., S. Jagabathula, D. Shah. 2013. A non-parametric approach to modeling choice with limited data. Management Science 59(2) 305 - 322.

Feldman, J. B., H. Topaloglu. 2014. Revenue management under the Markov chain choice model. Working Paper.

Gallego, G., H. Topaloglu. 2014. Constrained assortment optimization for the nested logit model. Management Science 60(10) 2583-2601.

Gary, M. R., D. S. Johnson. 1979. Computers and Intractability: A Guide to the Theory of NPCompleteness. W. H. Freeman and Co., San Francisco.

Honhon, D., S. Jonnalagedda, X. A. Pan. 2012. Optimal algorithms for assortment selection under ranking-based consumer choice models. Manufacturing $\&$ Service Operations Management 14(2) 279-289.

Jagabathula, S. 2016. Assortment optimization under general choice. Working Paper.

Kök, A., M. Fisher. 2007. Demand estimation and assortment optimization under substitution: Methodology and application. Operations Research 55(6) 1001-1021.

Li, G., P. Rusmevichientong. 2014. A greedy algorithm for the two-level nested logit model. Operations Research Letters 42(5) 319-324.

Li, G., P. Rusmevichientong, H. Topaloglu. 2015. The d-level nested logit model: Assortment and price optimization problems. Operations Research 63(2) 325-342.

Özer, Ö., R. Phillips. 2012. The Oxford Handbook of Pricing Management. Oxford University Press, Oxford, UK.

Paul, A., H. Topaloglu, J. Feldman. 2016. Assortment optimization for choosy customers. Working Paper.

Rusmevichientong, P., Z.-J. Max Shen, D. B. Shmoys. 2010. Dynamic assortment optimization with a multinomial logit choice model and capacity constraint. Operations Research $\mathbf{5 8}(6)$ $1666-1680$.

Rusmevichientong, P., D. Shmoys, C. Tong, H. Topaloglu. 2014. Assortment optimization under the multinomial logit model with random choice parameters. Production and Operations Management 23(11) 2023-2039.

Rusmevichientong, P., H. Topaloglu. 2012. Robust assortment optimization in revenue management under the multinomial logit choice model. Operations Research 60(4) 865-882. 
Sahin, O., R. Wang. 2016. The impact of consumer search cost on assortment planning and pricing. Working Paper.

Talluri, K., G. van Ryzin. 2004. Revenue management under a general discrete choice model of consumer behavior. Manage. Sci. 50(1) 15-33.

Train, K. E. 2009. Discrete Choice Methods with Simulation. Cambridge University Press.

Wang, R., Z. Wang. 2016. Consumer choice models with endogenous network effects. Management Science Forthcoming.

\section{Appendix}

Proof of Lemma 1. The lemma is trivial if $S=S^{\prime}$. In the following, we consider the case when $S \backslash$ $S^{\prime} \neq \phi$. Let $k \in S \backslash S^{\prime}$, we show that $\mathbf{R}\left(S, S^{\prime}\right) \leq \mathbf{R}\left(S \backslash\{k\}, S^{\prime}\right)$. Since $S^{\prime}$ is an optimal recommended set under $S$, we have

$$
\frac{\sum_{i \in S^{\prime}} r_{i} v_{i}}{\sum_{i \in S^{\prime}} v_{i}+v_{0}} \geq \frac{\sum_{i \in S^{\prime} \cup\{k\}} r_{i} v_{i}}{\sum_{i \in S^{\prime} \cup\{k\}} v_{i}+v_{0}} .
$$

By the assumption in the lemma, we must have $v_{k} \neq 0$, otherwise $S^{\prime \prime}=S^{\prime} \cup\{k\} \in$

$\arg \max _{R \subseteq S} \frac{\sum_{i \in R} r_{i} v_{i}}{\sum_{i \in R} v_{i}+v_{0}}$ which contradicts with the assumption. Combine (6) and that $v_{k} \neq 0$, we have $r_{k} \leq \frac{\sum_{i \in S^{\prime}} r_{i} v_{i}}{\sum_{i \in S^{\prime}} v_{i}+v_{0}}$. Thus $\mathbf{R}\left(S \backslash\{k\}, S^{\prime}\right)-\mathbf{R}\left(S, S^{\prime}\right)=\lambda_{k} \frac{\sum_{i \in S^{\prime}} r_{i} v_{i}}{\sum_{i \in S^{\prime}} v_{i}+v_{0}}-\lambda_{k} r_{k} \geq 0$. Repeating the above procedure, we obtain $\mathbf{R}\left(S, S^{\prime}\right) \leq \mathbf{R}\left(S^{\prime}, S^{\prime}\right)$ and the lemma holds.

Proof of Theorem 1. According to Lemma 1, there exists an optimal solution to the homogeneous MCST problem in which the recommended set is the entire set of available products. Therefore, we only need to consider the best $\mathbf{R}(S, S)$ in order to solve the homogeneous MCST problem. In the following, we show that for any assortment $S, \mathbf{R}(S, S)$ is equal to the revenue of assortment $S$ under the Markov chain choice model. To show that, it suffices to show that the choice probabilities of each product are identical for the two models. By (1), the choice probability for each $i \in S$ in the homogeneous MCST problem is $\operatorname{Pr}_{i}(S, S)=\lambda_{i}+\sum_{j: j \notin S} \lambda_{j} \frac{v_{i}}{\sum_{i \in S} v_{i}+v_{0}}$. Now we consider the choice probability under the Markov chain choice model. From Theorem 2.1 in Blanchet et al. (2016), the choice probability $\operatorname{Pr}_{i}^{M}(S)=\lambda_{i}+(\boldsymbol{\lambda}(\bar{S}))^{T}(\mathcal{I}-\rho(\bar{S}, \bar{S}))^{-1} \rho(\bar{S},\{i\})$, where $\bar{S}=\mathcal{N} \backslash S, \boldsymbol{\lambda}(\bar{S})$ is the vector of arrival probabilities in $\bar{S}, \mathcal{I}$ is the identity matrix and $\rho\left(S, S^{\prime}\right)$ is the transition matrix from products in $S$ to products in $S^{\prime}$. Note that $\rho(\bar{S},\{i\})=\left(v_{i}, \ldots, v_{i}\right)^{T}$ and $\sum_{i \in \mathcal{N} \cup\{0\}} v_{i}=1$, it can be verified that $(\mathcal{I}-\rho(\bar{S}, \bar{S}))^{-1} \rho(\bar{S},\{i\})=\left(v_{i} /\left(v_{0}+\sum_{i \in S} v_{i}\right), \ldots, v_{i} /\left(v_{0}+\sum_{i \in S} v_{i}\right)\right)^{T}$, and hence $\operatorname{Pr}_{i}^{M}(S)=\lambda_{i}+\sum_{j: j \notin S} \lambda_{j} \frac{v_{i}}{\sum_{i \in S} v_{i}+v_{0}}=\operatorname{Pr}_{i}(S, S)$. Thus the theorem holds.

Proof of Lemma 2. We first prove the first part. By simple algebra, we have

$$
\mathbf{R}(S \cup\{1\}, S \cup\{1\})
$$




$$
\begin{aligned}
= & \sum_{j \in S \cup\{1\}} \lambda_{j} r_{j}+\sum_{j \in \mathcal{N} \backslash(S \cup\{1\})} \lambda_{j} \frac{\sum_{i \in S \cup\{1\}} r_{i} v_{i}}{\sum_{i \in S \cup\{1\}} v_{i}+v_{0}} \\
= & \sum_{j \in S \cup\{1\}} \lambda_{j} r_{j}+\sum_{j \in \mathcal{N} \backslash(S \cup\{1\})} \lambda_{j} \frac{\sum_{i \in S} r_{i} v_{i}+r_{1} v_{1}}{\sum_{i \in S} v_{i}+v_{1}+v_{0}} \\
= & \sum_{j \in S \cup\{1\}} \lambda_{j} r_{j}+\sum_{j \in \mathcal{N} \backslash(S \cup\{1\})} \lambda_{j}\left(\frac{r_{1} v_{1}}{v_{1}+v_{0}}+\frac{\sum_{i \in S}\left(r_{i}-\frac{r_{1} v_{1}}{v_{1}+v_{0}}\right) v_{i}}{\sum_{i \in S} v_{i}+v_{1}+v_{0}}\right) \\
= & \lambda_{1} r_{1}+\sum_{j \in S} \lambda_{j} \frac{r_{1} v_{1}}{v_{1}+v_{0}}+\sum_{j \in \mathcal{N} \backslash(S \cup\{1\})} \lambda_{j} \frac{r_{1} v_{1}}{v_{1}+v_{0}} \\
& +\sum_{j \in S} \lambda_{j}\left(r_{j}-\frac{r_{1} v_{1}}{v_{1}+v_{0}}\right)+\sum_{j \in N \backslash(S \cup\{1\})} \lambda_{j} \frac{\sum_{i \in S}\left(r_{i}-\frac{r_{1} v_{1}}{v_{1}+v_{0}}\right) v_{i}}{\sum_{i \in S} v_{i}+v_{1}+v_{0}} \\
= & \lambda_{1} r_{1}+\sum_{j \in \mathcal{N} \backslash\{1\}} \lambda_{j} \frac{r_{1} v_{1}}{v_{1}+v_{0}}+\sum_{j \in S} \lambda_{j} r_{j}^{(1)}+\sum_{j \in(\mathcal{N} \backslash\{1\}) \backslash S} \lambda_{j} \frac{\sum_{i \in S} r_{i}^{(1)} v_{i}^{(1)}}{\sum_{i \in S} v_{i}^{(1)}+v_{0}^{(1)}} \\
= & \mathbf{R}(\{1\},\{1\})+\mathbf{R}^{(1)}(S, S) .
\end{aligned}
$$

Next, we prove the second part. We have when $r_{1} \geq 0$

$$
\begin{aligned}
\mathbf{R}(S \cup\{1\}, S \cup\{1\}) & =\sum_{j \in S \cup\{1\}} \lambda_{j} r_{j}+\sum_{j \notin S \cup\{1\}} \lambda_{j} \frac{\sum_{i \in S \cup\{1\}} r_{i} v_{i}}{\sum_{i \in S \cup\{1\}} v_{i}+v_{0}} \\
& =\sum_{j \in S} \lambda_{j} r_{j}+\sum_{j \notin S} \lambda_{j} \frac{\sum_{i \in S \cup\{1\}} r_{i} v_{i}}{\sum_{i \in S \cup\{1\}} v_{i}+v_{0}}+\lambda_{1} r_{1}-\lambda_{1} \frac{\sum_{i \in S \cup\{1\}} r_{i} v_{i}}{\sum_{i \in S \cup\{1\}} v_{i}+v_{0}} \\
& \geq \sum_{j \in S} \lambda_{j} r_{j}+\sum_{j \notin S} \lambda_{j} \frac{\sum_{i \in S} r_{i} v_{i}}{\sum_{i \in S} v_{i}+v_{0}}+\lambda_{1} r_{1}-\lambda_{1} \frac{\sum_{i \in S \cup\{1\}} r_{i} v_{i}}{\sum_{i \in S \cup\{1\}} v_{i}+v_{0}} \\
& =\mathbf{R}(S, S)+\lambda_{1}\left(r_{1}-\frac{\sum_{i \in S \cup\{1\}} r_{i} v_{i}}{\sum_{i \in S \cup\{1\}} v_{i}+v_{0}}\right) \geq \mathbf{R}(S, S),
\end{aligned}
$$

where the first inequality holds because when $r_{1} \geq 0, \frac{\sum_{i \in S \cup\{1\}} r_{i} v_{i}}{\sum_{i \in S \cup\{1\}} v_{i}+v_{0}} \geq \frac{\sum_{i \in S} r_{i} v_{i}}{\sum_{i \in S} v_{i}+v_{0}}$ and the last inequality holds because when $r_{1} \geq 0, r_{1} \geq \frac{\sum_{i \in S \cup\{1\}} r_{i} v_{i}}{\sum_{i \in S \cup\{1\}} v_{i}+v_{0}}$. Thus the lemma is proved.

Proof of Theorem 2. It is clear that the computational complexity of Algorithm 1 is $O(n)$. It remains to show that Step 7 actually computes the value of updated revenue in (3) in each iteration. We show this by induction. In fact, we will show a stronger statement that $r_{j}^{(i-1)}=r_{j}-\frac{R V_{i-1}}{V_{i-1}}$ gives the updated revenue for all $j \geq i$ in $i$ th iteration. (Note that products $1, \ldots, i-1$ have been selected before $i$ th iteration and there is no need to update them.) First, the result holds for $i=2$ by definition in (3). Now suppose the induction assumption holds in the $k$ th iteration, i.e., $r_{i}^{(k-1)}=r_{i}-\frac{R V_{k-1}}{V_{k-1}}$ for all $i \geq k$. Then, for the $(k+1)$ th iteration, by $(3)$, the updated revenue of product $i \geq k+1$ is 


$$
\begin{aligned}
r_{i}^{(k)} & =r_{i}^{(k-1)}-\frac{r_{k}^{(k-1)} v_{k}^{(k-1)}}{v_{0}^{(k-1)}+v_{k}} \\
& =r_{i}-\frac{R V_{k-1}}{V_{k-1}}-\frac{\left(r_{k}-\frac{R V_{k-1}}{V_{k-1}}\right) v_{k}}{\sum_{j=1}^{k-1} v_{j}+v_{k}} \\
& =r_{i}-r_{k}+\left(r_{k}-\frac{R V_{k-1}}{V_{k-1}}\right) \cdot \frac{V_{k-1}}{V_{k}} \\
& =r_{i}-\frac{r_{k} v_{k}}{V_{k}}-\frac{R V_{k-1}}{V_{k}} \\
& =r_{i}-\frac{R V_{k}}{V_{k}}
\end{aligned}
$$

where $v_{0}^{(k-1)}=\sum_{j=1}^{k-1} v_{j}$ and $v_{k}^{(k-1)}=v_{k}$. Therefore, the induction assumption holds for $i=k+1$. Thus, by the induction principle and Lemma 2, Theorem 2 holds.

Proof of Theorem 3. We prove the theorem by a reduction from the unweighted independent set problem, which is known to be NP-Hard in the strong sense (Gary and Johnson 1979):

Independent Set Problem: Given a graph $G=(V, E)$, and an integer $0<k \leq n$, the problem is to decide if $G$ contains a subset $I \subseteq V$ such that $|I| \geq k$ and no two vertices in $I$ are joined by an edge in $E$.

Given an instance of the independent set problem with $G=(V, E)$, we construct an instance of the MCST problem with $m+n+1$ products, where $n=|V|$ and $m=|E|$. For ease of exposition, we assume that the arrival probabilities are unnormalized, i.e., they might be greater than 1 . This is without loss of generality since we can normalize them by dividing their sum. The products are divided into three classes. For each edge $e \in E$, we create a product, called the edge product, each has arrival probability 1 and revenue 0 . For each vertex $i \in V$, we create a product, called the vertex product, each has arrival probability $\frac{1}{n+k}$ and revenue 1 . There is also a dummy product $d$, which has arrival probability 0 and revenue 2 . The transition weights between the products are defined as follows. The edge products can transit to the vertex products. Specifically, if $e=(i, j)$, then the transition weights are $v_{e i}=v_{e j}=1 / 2$, and the weights are 0 otherwise (including the no-purchase option). Each vertex product can only transit to the dummy product, and the weights are $v_{i d}=1$ and $v_{i 0}=0$ for any $i \in V$. It can be seen that in the construction, the largest value is polynomially bounded by the size of the input instance (thus it is a polynomial-time reduction). We illustrate the construction in Figure 2.

In the following, we show that there exists an independent set of size at least $k$ if and only if the optimal assortment to the MCST problem has a revenue of at least $m+1$. If this holds, then by the strong NP-Hardness of the independent set problem, we know that the MCST problem is 


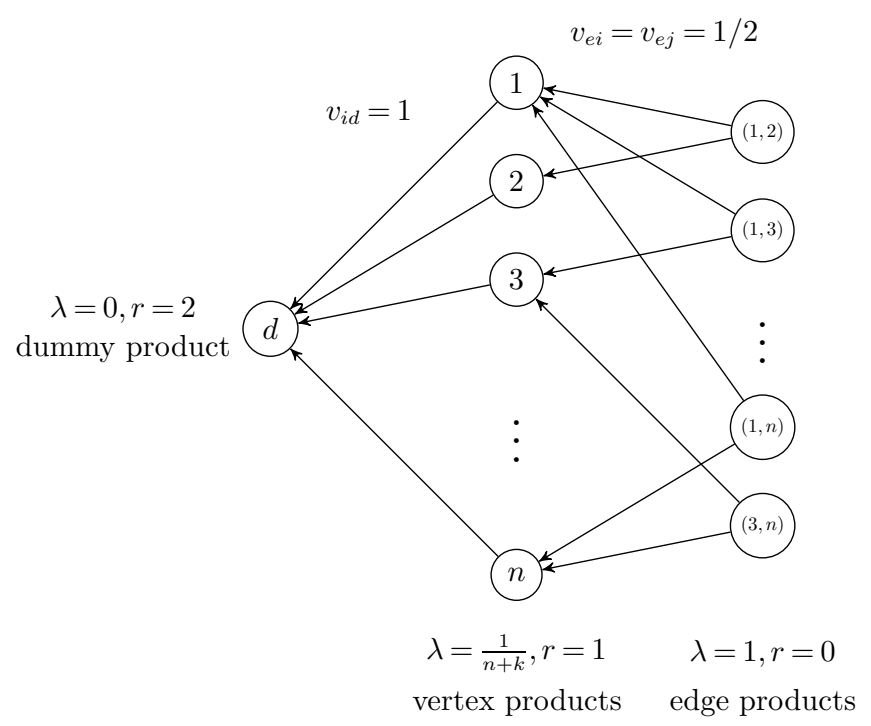

Figure 2 Illustration of the Reduction from the Independent Set Problem

also strongly NP-Hard (and note that in the construction each product only transits to at most two products, thus the theorem holds).

First, we prove that if there exists an independent set of size $k$, then there exists an assortment in the MCST problem that gives a revenue of at least $m+1$. By the construction of the instance, it is obvious that an optimal assortment must include the dummy product which has zero arrival probability, but not any edge product which has zero revenue. Let $U$ be the subset of vertex products that are selected into the assortment, and $\bar{U}$ be those that are not in the assortment. Denote $\mathbf{R}_{E}$ as the revenue of the edge products (obtained by transiting to the products in $U$ ), $\mathbf{R}_{U}$ as the revenue of products $U$ and $\mathbf{R}_{\bar{U}}$ as the revenue of products $\bar{U}$ (obtained by transiting to the dummy product). Note that the revenue of the dummy product itself is 0 since its arrival probability is 0 . Thus, the total revenue can be represented as $\mathbf{R}=\mathbf{R}_{E}+\mathbf{R}_{U}+\mathbf{R}_{\bar{U}}$.

Suppose that there exists an independent set $I$ of size at most $k$. We consider the assortment by selecting the vertex product into $U$ if and only if its corresponding vertex is not in the independent set $I$. For each edge product, the revenue is 1 if at least one of its endpoints is in $U$ (regardless of which one or both), and the revenue is 0 if none of its endpoints is in $U$ (recall we assumed in the remark after (2) that the revenue is 0 in such cases, and such assumption is without loss of generality because we can choose the transition probability to the no-purchase option as an infinitesimal value instead of 0 in the construction). Since $I$ is an independent set, at least one of the endpoint is not in $I$ for each edge, thus it follows that $\mathbf{R}_{E}=m$. Furthermore, the revenue of all the selected vertex products is $\mathbf{R}_{U}=\frac{|U|}{n+k}=\frac{n-|I|}{n+k}$, and the remaining revenue is $\mathbf{R}_{\bar{U}}=\frac{2|I|}{n+k}$. Therefore, there exists an assortment which has revenue $\mathbf{R}=\mathbf{R}_{E}+\mathbf{R}_{U}+\mathbf{R}_{\bar{U}}=m+\frac{n+|I|}{n+k} \geq m+1$ since $|I| \geq k$. 
Now we prove the other direction. Suppose that there exists an assortment which has revenue at least $\mathbf{R} \geq m+1$. We select the vertices into the independent set $I$ if and only if the corresponding vertex product does not belong to the assortment $U$. We first claim that $\mathbf{R}_{E} \geq m$, which implies $\mathbf{R}_{E}=m$ since each edge product can contribute to the revenue by at most 1 . Assume that $\mathbf{R}_{E} \leq m-1$. By the arguments above, we have $\mathbf{R}_{U}+\mathbf{R}_{\bar{U}}=\frac{n+|I|}{n+k}$. Note that $k>0$ and $|I| \leq n$, it follows that $\mathbf{R}=\mathbf{R}_{E}+\mathbf{R}_{U}+\mathbf{R}_{\bar{U}} \leq m-1+\frac{n+|I|}{n+k}<m-1+\frac{2 n}{n}=m+1$, which contradicts with the assumption that $\mathbf{R} \geq m+1$. Therefore $\mathbf{R}_{E}=m$ and each edge has at least one endpoint in $U$. In other words, the vertex set $I$ constitutes an independent set. Furthermore, we have $\mathbf{R}_{U}+\mathbf{R}_{\bar{U}}=\frac{n+|I|}{n+k} \geq 1$, and thus $|I| \geq k$. Therefore, the instance has an independent set with size at least $k$. Thus, the theorem holds.

Proof of Theorem 4. The correctness of the DP directly follows from the recursion formula. Now we briefly discuss the computational complexity. We note that in the dynamic program, the state space is of size $O(n)$. Moreover, the recursion formula requires going through each edge once, which is also of size $O(n)$ because each product can only transit to at most one other product. Therefore, the computational complexity of the DP is $O(n)$.

Proof of Theorem 6. Let $S^{*}$ be the optimal assortment under the MCST model with optimal value OPT and the best recommend sets $R_{j}^{S^{*}}$ for all $j \notin S^{*}$. Let $S_{t}=\{1, \ldots, t\}$ be the optimal revenue-ordered assortment with revenue $\mathbf{R}^{r o}$ and the best recommended sets $R_{j}^{S_{t}}$ for $j>t$. First, we have

$$
\begin{aligned}
\mathrm{OPT} & =\sum_{i=1}^{n} r_{i} \operatorname{Pr}_{i}\left(S^{*}, R_{j}^{S^{*}}\right)=\sum_{t=1}^{n}\left(r_{t}-r_{t+1}\right) \sum_{i=1}^{t} \operatorname{Pr}_{i}\left(S^{*}, R_{j}^{S^{*}}\right) \\
& =\sum_{t=1}^{n}\left(r_{t}-r_{t+1}\right) \sum_{i \in S^{*} \cap S_{t}} \operatorname{Pr}_{i}\left(S^{*}, R_{j}^{S^{*}}\right)=\sum_{t=1}^{n} \frac{r_{t}-r_{t+1}}{r_{t}} \sum_{i \in S^{*} \cap S_{t}} r_{t} \operatorname{Pr}_{i}\left(S^{*}, R_{j}^{S^{*}}\right)
\end{aligned}
$$

Note that we let $r_{n+1}=0$ and the equalities hold since $\operatorname{Pr}_{i}\left(S^{*}, R_{j}^{S^{*}}\right)=0$ if $i \notin S^{*}$. From (1), we have

$$
\begin{aligned}
& \sum_{i \in S^{*} \cap S_{t}} r_{t} \operatorname{Pr}_{i}\left(S^{*}, R_{j}^{S^{*}}\right) \\
= & \sum_{i \in S^{*} \cap S_{t}} \lambda_{i} r_{t}+\sum_{i \in S^{*} \cap S_{t}} \sum_{j: j \notin S, i \in R_{j}^{S^{*}}} \lambda_{j} r_{t} \frac{v_{j i}}{\sum_{i \in R_{j} S^{*}} v_{j i}+v_{j 0}} \\
= & \sum_{i \in S^{*} \cap S_{t}} \lambda_{i} r_{t}+\sum_{j: j \notin S^{*}} \lambda_{j} r_{t} \frac{\sum_{i \in R_{j}^{S^{*}} \cap S_{t}} v_{j i}}{\sum_{i \in R_{j}^{S^{*}}} v_{j i}+v_{j 0}} \\
\leq & \sum_{i \in S^{*} \cap S_{t}} \lambda_{i} r_{t}+\sum_{j: j \notin S^{*} \cap S_{t}} \lambda_{j} r_{t} \frac{\sum_{i \in R_{j}^{S^{*}} \cap S_{t}} v_{j i}}{\sum_{i \in R_{j}^{S^{*}} \cap S_{t}} v_{j i}+v_{j 0}}
\end{aligned}
$$




$$
\begin{aligned}
& =\sum_{i \in S^{*} \cap S_{t}} \lambda_{i} r_{t}+\sum_{j: j \notin S_{t}} \lambda_{j} r_{t} \frac{\sum_{i \in R_{j}^{S^{*}} \cap S_{t}} v_{j i}}{\sum_{i \in R_{j}^{S^{*}} \cap S_{t}} v_{j i}+v_{j 0}}+\sum_{j: j \in S_{t} \backslash S^{*}} \lambda_{j} r_{t} \frac{\sum_{i \in R_{j}^{S^{*}} \cap S_{t}} v_{j i}}{\sum_{i \in R_{j}^{S *} \cap S_{t}} v_{j i}+v_{j 0}} \\
& \leq \sum_{i \in S^{*} \cap S_{t}} \lambda_{i} r_{i}+\sum_{j: j \notin S_{t}} \lambda_{j} \frac{\sum_{i \in R_{j}^{S *} \cap S_{t}} v_{j i} r_{i}}{\sum_{i \in R_{j}^{S^{*}} \cap S_{t}} v_{j i}+v_{j 0}}+\sum_{j: j \in S_{t} \backslash S^{*}} \lambda_{j} r_{j} \frac{\sum_{i \in R_{j}^{S *} \cap S_{t}} v_{j i}}{\sum_{i \in R_{j}^{S^{*}} \cap S_{t}} v_{j i}+v_{j 0}} \\
& \leq \sum_{i \in S^{*} \cap S_{t}} \lambda_{i} r_{i}+\sum_{j: j \notin S_{t}} \lambda_{j} \frac{\sum_{i \in R_{j}^{S t}} v_{j i} r_{i}}{\sum_{i \in R_{j}^{S_{t}}} v_{j i}+v_{j 0}}+\sum_{j: j \in S_{t} \backslash S^{*}} \lambda_{j} r_{j} \\
& =\sum_{i \in S_{t}} \lambda_{i} r_{i}+\sum_{j: j \notin S_{t}} \lambda_{j} \frac{\sum_{i \in R_{j}^{S t}} v_{j i} r_{i}}{\sum_{i \in R_{j}^{S_{t}} v_{j i}+v_{j 0}}}=\sum_{i \in S_{t}} r_{i} \operatorname{Pr}_{i}\left(S_{t}, R_{j}^{S_{t}}\right)=\mathbf{R}^{r o},
\end{aligned}
$$

where (8) holds since $S^{*} \cap S_{t} \subseteq S^{*}$ and $R_{j}^{S^{*}} \cap S_{t} \subseteq R_{j}^{S^{*}}$, (9) holds since $r_{t} \leq r_{i}$ for each $i \in S_{t}$, (10)

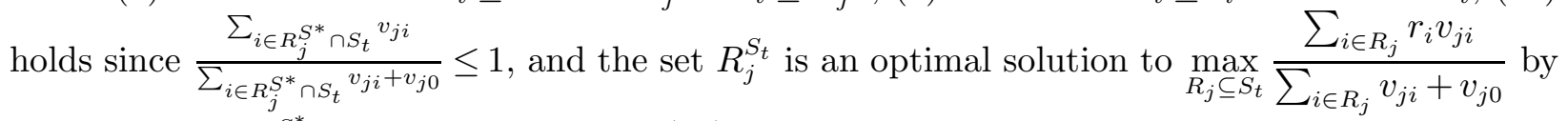
definition and $R_{j}^{S^{*}} \cap S_{t} \subseteq S_{t}$. Note that (11) is the revenue of the assortment $S_{t}$ under its best recommended set. Plugging it into (7), and the revenue-ordered assortment returns the assortment with maximum revenue among all $S_{t}$, we obtain

$$
\mathrm{OPT} \leq \sum_{t=1}^{n} \frac{r_{t}-r_{t+1}}{r_{t}} \mathbf{R}^{r o} \leq\left(1+\sum_{t=1}^{n-1} \int_{r_{t+1}}^{r_{t}} \frac{1}{x} d x\right) \mathbf{R}^{r o}=\left(1+\log \frac{r_{1}}{r_{n}}\right) \mathbf{R}^{r o} .
$$

Furthermore, let $\tilde{r}_{1}, \ldots, \tilde{r}_{d}$ be the distinct revenue values sorted in decreasing order. Similarly, we can obtain from (11) that

$$
\mathrm{OPT}=\sum_{i \in S^{*}} r_{i} \operatorname{Pr}_{i}\left(S^{*}, R_{j}^{S^{*}}\right)=\sum_{t=1}^{d} \tilde{r}_{t} \sum_{i \in S^{*}, r_{i}=\tilde{r}_{t}} \operatorname{Pr}_{i}\left(S^{*}, R_{j}^{S^{*}}\right) \leq \sum_{t=1}^{d} \tilde{r}_{t} \sum_{i \in S^{*} \cap S_{t}} \operatorname{Pr}_{i}\left(S^{*}, R_{j}^{S^{*}}\right) \leq d \mathbf{R}^{r o} .
$$

Combining (12) and (13), we obtain that the revenue of the best revenue-ordered assortment achieves at least $\max \left\{\frac{1}{d}, \frac{1}{1+\log \frac{r_{\max }}{r_{\min }}}\right\}$ fraction of the optimal revenue.

Finally, we show that the performance bound is tight in the sense that we can find an instance such that the revenue-ordered assortment only achieves $O\left(\frac{1}{d}\right)$ and $O\left(\frac{1}{1+\log \frac{r_{\max }}{r_{\min }}}\right)$ fraction of the optimal revenue respectively. Let $k \geq 1, \epsilon \in(0,1)$ and the number of products be $n=2 k-1$. For ease of exposition, we denote the products as $k$ classes, where each class has two products except for the $k$ th class which has only one product. The revenue of product in class $i$ is $\epsilon^{-i+1}$, for $i=1, \ldots, k$. For $i=1, \ldots, k-1$, one product in class $i$ is denoted as product $p_{i}^{1}$, which has arrival probability $\epsilon^{i}$; and the other product is denoted as $p_{i}^{0}$, each has arrival probability 0 , except for the product $p_{1}^{0}$ which has arrival probability $1-\sum_{j=1}^{k-1} \epsilon^{j}$. The class $k$ has only product $p_{k}^{0}$, which has arrival probability 0 . For product $p_{i}^{1}, i=1, \ldots, k-1$, the transition probability to the product $p_{i+1}^{0}$ is 1 , and the transition probabilities to other products are 0 (including to the no-purchase option). For product $p_{i}^{0}, i=1, \ldots, k$, the transition probability to the no-purchase option is 1 , and the transition probabilities to other products are 0 . In other words, except for the transitions to the no-purchase 


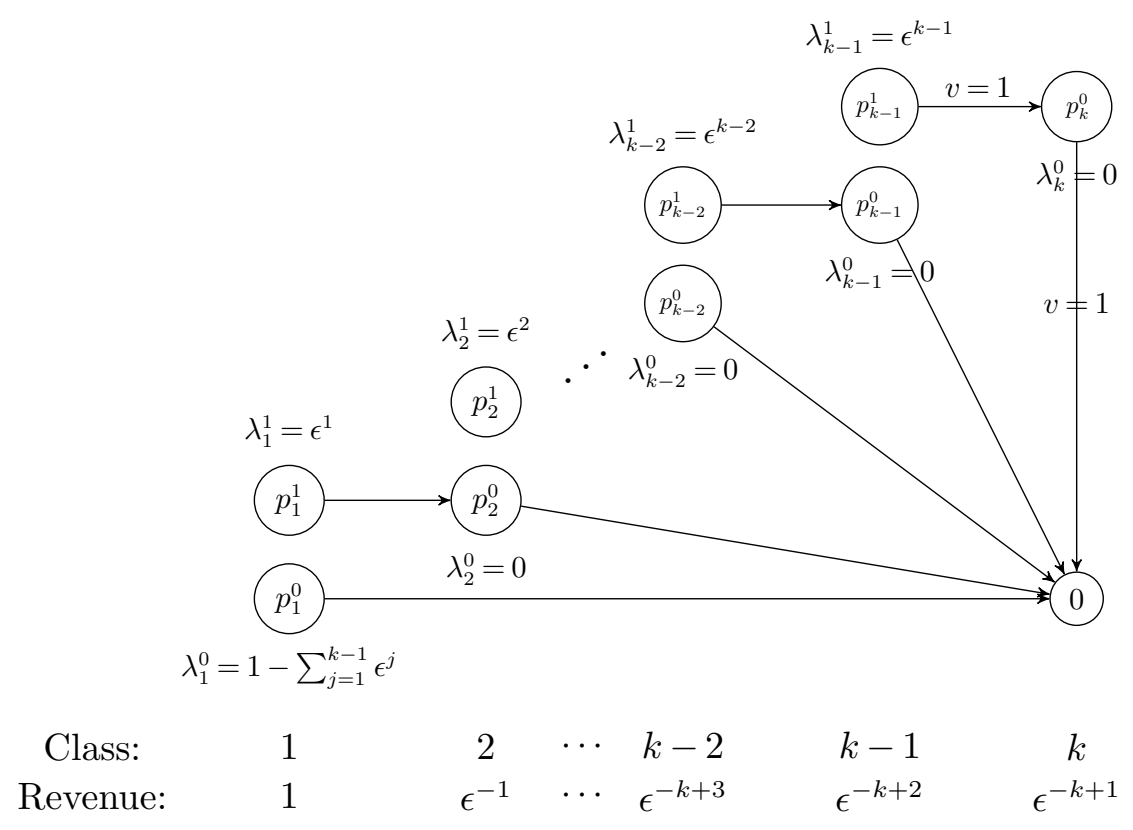

\section{Figure 3 Tight Example of Revenue-Ordered Assortment}

option, the only possible transition is from an unavailable product $p_{i}^{1}$ to an available product $p_{i+1}^{0}$. The construction of the case is illustrated in Figure 3.

We first discuss the bound $O(1 / d)$. Note that this instance has exactly $k$ distinct revenue values, and within each class, there are three possibilities to choose the products. Let $S_{t}$ be the revenue-ordered assortment that all the products in classes $k, k-1, \ldots, k-t+1$ are selected, $\forall t=1, \ldots, k$, and $S_{t}^{0}=S_{t} \backslash\left\{p_{k-t+1}^{1}\right\}$ and $S_{t}^{1}=S_{t} \backslash\left\{p_{k-t+1}^{0}\right\}$ be the other two types of revenue-ordered assortments, $\forall t=2, \ldots, k$. It can be computed that for $t=1, \ldots, k-1$, the revenue of assortment $S_{t}$ is $\sum_{j=k-t+1}^{k-1} \epsilon^{j} \cdot \epsilon^{-j+1}+\epsilon^{k-t} \cdot \epsilon^{-k+t}=1+(t-1) \epsilon$, where the first term in the left-hand side corresponds to the $p_{j}^{1}$ products for $j=k-t+1, \ldots, k-1$ selected in $S_{t}$, and the second term corresponds to the unavailable product $p_{k-t}^{1}$ transiting to the product $p_{k-t+1}^{0}$ in $S_{t}$ (the revenue for selecting $p_{j}^{0}$ products is 0 since the arrival probability is 0 ); the revenue of the last revenue-ordered assortment $S_{k}$ is $\sum_{j=1}^{k-1} \epsilon^{j} \cdot \epsilon^{-j+1}+\left(1-\sum_{j=1}^{k-1} \epsilon^{j}\right) \cdot \epsilon^{-1+1}=1+(k-2) \epsilon-\sum_{j=2}^{k-1} \epsilon^{j}$. For $t=2, \ldots, k$, the revenue of assortment $S_{t}^{1}$ is $\sum_{j=k-t+1}^{k-1} \epsilon^{j} \cdot \epsilon^{-j+1}=(t-1) \epsilon$, and the revenue of assortment $S_{t}^{0}$ is $\sum_{j=k-t+2}^{k-1} \epsilon^{j} \cdot \epsilon^{-j+1}+\epsilon^{k-t+1} \cdot \epsilon^{-k+t+1}+\epsilon^{k-t} \cdot \epsilon^{-k+t}=2+(t-2) \epsilon$, except that $S_{k}^{0}=2+(k-2) \epsilon-\sum_{j=2}^{k-1} \epsilon^{j}$. If we select all the $p_{i}^{0}$ products for $i=1, \ldots, k$ but not the $p_{i}^{1}$ products, then the revenue is $\sum_{j=2}^{k} \epsilon^{j-1} \cdot \epsilon^{-j+1}+\left(1-\sum_{j=1}^{k-1} \epsilon^{j}\right) \cdot \epsilon^{-1+1}=k-\sum_{j=1}^{k-1} \epsilon^{j}$. Therefore, the ratio of the revenue of the revenue-ordered assortment and the optimal assortment is $2 / k+O(\epsilon)$, it goes to $2 / k=O(1 / d)$ when $\epsilon$ goes to 0 . Moreover, we can see that $\log \left(\frac{r_{\max }}{r_{\min }}\right)=(k-1) \log (1 / \epsilon)$. Thus, as $k$ goes to infinity, the second bound is also tight. 
Proof of Theorem 7. Given any fixed $\boldsymbol{x} \in\{0,1\}^{n}$, we prove that the remaining optimization problem in (5) and (4) are the same. First, fixing $\boldsymbol{x}$, we show that (4) is equivalent to the problem

$$
\begin{array}{lll}
\max & \sum_{j=1}^{n} \lambda_{j}\left(1-x_{j}\right) \sum_{i=1}^{n}\left(r_{i}-z_{j}^{*}\right) \frac{v_{j i}}{v_{j 0}} y_{j i} & \\
\text { s.t. } & y_{j i} \leq x_{i}, & \forall i, j=1, \ldots, n, \\
& y_{j i} \leq 1-x_{j}, & \forall i, j=1, \ldots, n, \\
& 0 \leq y_{j i} \leq 1, & \forall i, j=1, \ldots, n,
\end{array}
$$

where $\boldsymbol{y}^{*}$ is an optimal solution to (4) (while fixing $\boldsymbol{x}$ ) and $z_{j}^{*}=\sum_{i=1}^{n} r_{i} v_{j i} y_{j i}^{*} /\left(\sum_{i=1}^{n} v_{j i} y_{j i}^{*}+v_{j 0}\right)$. By the definition of $\boldsymbol{y}^{*}$ and $z_{j}^{*}$, we have $\sum_{i=1}^{n} r_{i} v_{j i} y_{j i}^{*}=z_{j}^{*}\left(\sum_{i=1}^{n} v_{j i} y_{j i}^{*}+v_{j 0}\right)$, that is, $\sum_{i=1}^{n}\left(r_{i}-\right.$ $\left.z_{j}^{*}\right) \frac{v_{j i}}{v_{j 0}} y_{j i}^{*}=z_{j}^{*}$. Therefore, the optimal value to (14) is at least as large as the optimal value to (4). Now let $\tilde{\boldsymbol{y}}$ be an optimal solution to (14). Note that (14) is a linear programming problem. We claim that the constraint matrix of $\boldsymbol{y}$ formed by (14b) and (14c) is a totally unimodular matrix with dimension $2 n^{2} \times n^{2}$, since there is exactly one nonzero entry in each row. Therefore, we can

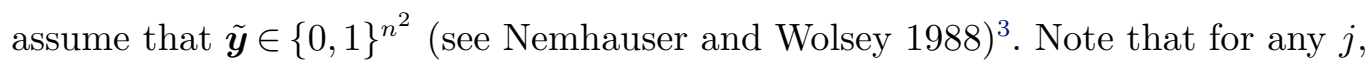

$$
\lambda_{j}\left(1-x_{j}\right) z_{j}^{*}=\lambda_{j}\left(1-x_{j}\right) \frac{\sum_{i=1}^{n} r_{i} v_{j i} y_{j i}^{*}}{\sum_{i=1}^{n} v_{j i} y_{j i}^{*}+v_{j 0}} \geq \lambda_{j}\left(1-x_{j}\right) \frac{\sum_{i=1}^{n} r_{i} v_{j i} \tilde{y}_{j i}}{\sum_{i=1}^{n} v_{j i} \tilde{y}_{j i}+v_{j 0}} .
$$

By rearranging terms, we get

$$
\lambda_{j}\left(1-x_{j}\right) z_{j}^{*} \geq \lambda_{j}\left(1-x_{j}\right) \sum_{i=1}^{n}\left(r_{i}-z_{j}^{*}\right) \frac{v_{j i}}{v_{j 0}} \tilde{y}_{j i},
$$

thus $\sum_{j=1}^{n} \lambda_{j}\left(1-x_{j}\right) z_{j}^{*} \geq \sum_{j=1}^{n} \lambda_{j}\left(1-x_{j}\right) \sum_{i=1}^{n}\left(r_{i}-z_{j}^{*}\right) \frac{v_{j i}}{v_{j 0}} \tilde{y}_{j i}$. Therefore, the optimal value to (4) is at least as large as the optimal value to (14), i.e., the optimal values to the two problems are the same. Thus, for any given $\boldsymbol{x}$ and given an optimal solution to one of the two problems, we can construct a feasible solution to the other which has the same objective value, which implies that (4) and (14) are equivalent.

Next we show that (14) and (5) are equivalent. On one hand, let $\boldsymbol{y}^{*}$ be an optimal solution to (14), and $\operatorname{OPT}\left(\boldsymbol{y}^{*}\right)$ be the optimal value. We construct the solution $z_{j i}=v_{j i} y_{j i}^{*}\left(1-x_{j}\right) /\left(\sum_{i=1}^{n} v_{j i} y_{j i}^{*}+v_{j 0}\right)$ and $z_{j 0}=v_{j 0}\left(1-x_{j}\right) /\left(\sum_{i=1}^{n} v_{j i} y_{j i}^{*}+v_{j 0}\right), \forall i, j=1, \ldots, n$ to problem (5). It can be verified that the solution $\boldsymbol{z}$ is feasible to (5). In particular, if $x_{i}=0$, then $y_{j i}^{*}=0$ from constraint (14b) and we have $z_{j i}=0 \leq x_{i}$; otherwise, by the above definition and $x_{j} \in\{0,1\}, z_{j i} \leq 1-x_{j} \leq 1=x_{i}$, therefore constraint (5b) is always satisfied. Constraint (5c) is clearly satisfied from the definition of $\boldsymbol{z}$. Moreover, we have $\frac{z_{j i}}{z_{j 0}}=\frac{v_{j i} y_{j i}^{*}}{v_{j 0}}$, i.e., $z_{j i}=\frac{v_{j i}}{v_{j 0}} z_{j 0} y_{j i}^{*}$, it follows that $z_{j i} \leq \frac{v_{j i}}{v_{j 0}} z_{j 0}$ since $y_{j i}^{*} \leq 1$ and thus constraint

${ }^{3}$ G. L. Nemhauser and L. A. Wolsey. 1988. Integer and Combinatorial Optimization. Wiley-Interscience, New York, NY, USA. 
$(5 \mathrm{~d})$ is satisfied. Note that for each $j=1, \ldots, n, \sum_{i=1}^{n} r_{i} z_{j i}=\left(1-x_{j}\right) \sum_{i=1}^{n} r_{i} v_{j i} y_{j i}^{*} /\left(\sum_{i=1}^{n} v_{j i} y_{j i}^{*}+\right.$ $\left.v_{j 0}\right)$, Thus it implies that the objective value of $\boldsymbol{z}$ to (5) is at least $\operatorname{OPT}\left(\boldsymbol{y}^{*}\right)$. Therefore, the optimal value to (5) is at least as large as the optimal value to (14).

On the other hand, let $\tilde{\boldsymbol{z}}$ be an optimal solution to (5), and $\operatorname{OPT}(\tilde{\boldsymbol{z}})$ be the optimal value. We construct the solution $y_{j i}=v_{j 0} \tilde{z}_{j i} /\left(v_{j i} \tilde{z}_{j 0}\right), \forall i, j=1, \ldots, n$. Now we discuss its feasibility to (14). By constraint $(5 \mathrm{~d})$ and the construction of $y_{j i}$, we have $0 \leq y_{j i} \leq 1$. For each $\tilde{z}_{j i}$, if $\tilde{z}_{j i}=0$, then $y_{j i}=0$, and constraints (14b) and (14c) must be satisfied since $\boldsymbol{x} \in\{0,1\}$; otherwise, $\tilde{z}_{j i}>0$ and it implies that $x_{i}=1$ and $1-x_{j}=1$, thus constraints (14b) and (14c) are also satisfied since $y_{j i} \leq 1$ as mentioned before. Note that in the objective function (14a), the value $z_{j}^{*} \geq \sum_{i=1}^{n} r_{i} v_{j i} y_{j i} /\left(\sum_{i=1}^{n} v_{j i} y_{j i}+\right.$ $\left.v_{j 0}\right)$ by the definition of $z_{j}^{*}$. By rearranging terms, we get $v_{j 0} z_{j}^{*} \geq \sum_{i=1}^{n} r_{i} v_{j i} y_{j i}-z_{j}^{*} \sum_{i=1}^{n} v_{j i} y_{j i}=$ $\sum_{i=1}^{n} r_{i} v_{j 0} \tilde{z}_{j i} / \tilde{z}_{j 0}-z_{j}^{*} \sum_{i=1}^{n} v_{j 0} \tilde{z}_{j i} / \tilde{z}_{j 0}$, or equivalently, $z_{j}^{*} \geq \sum_{i=1}^{n} r_{i} \tilde{z}_{j i} / \tilde{z}_{j 0}-z_{j}^{*} \sum_{i=1}^{n} \tilde{z}_{j i} / \tilde{z}_{j 0}$. Since (4) and (14) are equivalent and $\operatorname{OPT}(\tilde{\boldsymbol{z}}) \geq \operatorname{OPT}\left(\boldsymbol{y}^{*}\right)=\sum_{j=1}^{n} \lambda_{j} r_{j} x_{j}+\sum_{j=1}^{n} \lambda_{j}\left(1-x_{j}\right) z_{j}^{*}$, the optimal solution to (14) is at least

$$
\begin{aligned}
\sum_{j=1}^{n} \lambda_{j} r_{j} x_{j}+\sum_{j=1}^{n} \lambda_{j}\left(1-x_{j}\right) & z_{j}^{*} \geq \sum_{j=1}^{n} \lambda_{j} r_{j} x_{j}+\sum_{j=1}^{n} \lambda_{j}\left(1-x_{j}\right)\left(\sum_{i=1}^{n} r_{i} \frac{\tilde{z}_{j i}}{\tilde{z}_{j 0}}-z_{j}^{*} \sum_{i=1}^{n} \frac{\tilde{z}_{j i}}{\tilde{z}_{j 0}}\right) \\
& \geq \sum_{j=1}^{n} \lambda_{j} r_{j} x_{j}+\sum_{j=1}^{n} \lambda_{j}\left(1-x_{j}\right) \sum_{i=1}^{n} r_{i} \frac{\tilde{z}_{j i}}{\tilde{z}_{j 0}}-\lambda_{j} \sum_{i=1}^{n} r_{i} \tilde{z}_{j i} \sum_{i=1}^{n} \frac{\tilde{z}_{j i}}{\tilde{z}_{j 0}} \\
& =\sum_{j=1}^{n} \lambda_{j} r_{j} x_{j}+\sum_{j=1}^{n} \lambda_{j}\left(1-x_{j}\right) \sum_{i=1}^{n} \tilde{z}_{j i}=\operatorname{OPT}(\tilde{\boldsymbol{z}}),
\end{aligned}
$$

where the second last equality follows from constraint (5c). Therefore, the optimal value to (14) is at least as large as the optimal value to (5), and thus the two problems are equivalent. Recall that the above arguments hold for arbitrary $\boldsymbol{x} \in\{0,1\}^{n}$, thus the theorem holds. 